This document is confidential and is proprietary to the American Chemical Society and its authors. Do not copy or disclose without written permission. If you have received this item in error, notify the sender and delete all copies.

\title{
Structural Basis for the Enantioselectivity of Esterase Est- Y29 toward (S)-Ketoprofen
}

\begin{tabular}{|c|c|}
\hline Journal: & ACS Catalysis \\
\hline Manuscript ID & CS-2018-02797v.R1 \\
\hline Manuscript Type: & Article \\
\hline $\begin{array}{r}\text { Date Submitted by the } \\
\text { Author: }\end{array}$ & 03-Dec-2018 \\
\hline Complete List of Authors: & $\begin{array}{l}\text { Ngo, Tri; Sungkyunkwan University School of Medicine } \\
\text { Oh, Changsuk; Sungkyunkwan University School of Medicine, Dept. of } \\
\text { Molecular Cell Biology } \\
\text { Mizar, Pushpak; University of Southampton, Chemistry } \\
\text { Baek, Minkyung; Seoul National University } \\
\text { Park, Kwang-su; Konkuk University, Dept. of Bioscience \& Biotechnology } \\
\text { Nguyen, Lan; Sungkyunkwan University School of Medicine, Molecular } \\
\text { Cell Biology } \\
\text { Byeon, Huimyoung; Sungkyunkwan University - Suwon Campus, Energy } \\
\text { Science } \\
\text { Yoon, Sangyoung; Ajou University } \\
\text { Ryu, Yeonwoo; Ajou University } \\
\text { Ryu, Bum Han; Sungkyunkwan University School of Medicine, Molecular } \\
\text { Cell Biology } \\
\text { Kim, T. Doohun ; Sookmyung Women's University } \\
\text { Yang, Jung Woon; Sungkyunkwan University - Suwon Campus, Energy } \\
\text { Science } \\
\text { Seok, Chaok; Seoul National University, Department of Chemistry } \\
\text { Lee, Seung Seo; University of Southampton, Chemistry } \\
\text { Kim, Kyeong; Sungkyunkwan University School of Medicine, Molecular } \\
\text { Cell Biology }\end{array}$ \\
\hline
\end{tabular}

\section{SCHOLARONE ${ }^{m}$ Manuscripts}


5 Tri Duc $\mathrm{Ngo}^{1 \$}$, Changsuk $\mathrm{Oh}^{1 \$}$, Pushpak Mizar ${ }^{2}$, Minkyung Baek ${ }^{3}$, Kwang-su Park ${ }^{1}$, Lan 6 Nguyen ${ }^{1}$, Huimyoung Byeon ${ }^{4}$, Sangyoung Yoon ${ }^{5}$, Yeonwoo Ryu ${ }^{5}$, Bum Han Ryu ${ }^{1}$, T. Doohun $7 \mathrm{Kim}^{6}$, Jung Woon Yang ${ }^{4}$, Chaok Seok ${ }^{3}$, Seung Seo Lee ${ }^{2}$, Kyeong Kyu Kim ${ }^{1 *}$

$9{ }^{1}$ Department of Molecular Cell Biology, Sungkyunkwan University School of Medicine, 10 Suwon, 16419, Republic of Korea; ${ }^{2}$ Chemistry, Highfield Campus, University of Southampton, 11 Southampton, SO17 1BJ, UK. ${ }^{3}$ Department of Chemistry, College of Natural Sciences, Seoul 12 National University, Seoul, 08826, Republic of Korea; ${ }^{4}$ Department of Energy, Sungkyunkwan 13 University, Suwon, 16419, Republic of Korea; ${ }^{5}$ Department of Applied Chemistry \& 14 Biological Engineering, Ajou University, Suwon, 16499, Republic of Korea; ${ }^{6}$ Department of 15 Chemistry, College of Natural Sciences, Sookmyung Women's University, Seoul, 04310, 16 Republic of Korea 


\section{Abstract}

19 The thermostable esterase Est-Y29, belonging to the family VIII lipolytic esterase isolated 20 from metagenomes extracted from the topsoil in Republic of Korea, was identified as a 21 promising catalyst for the production of $(S)$-ketoprofen, an important nonsteroidal anti22 inflammatory drug (NSAID). For industrial applications, the enantioselectivity of the enzyme 23 towards the $S$-enantiomer of the racemic ketoprofen ester substrate needs to be improved. To 24 understand the structural basis of Est-Y29 enantioselectivity, which is necessary for the rational 25 design of an enzyme with enhanced enantioselectivity, we solved the crystal structures of Est26 Y29 bound to $(S)$-ketoprofen at $1.69 \AA$ resolution. Structural analyses revealed that the $S$ 27 enantiomer can be stabilized by a $\pi$-interaction between the methyl substituent at the chiral 28 carbon of the ligand and the aromatic pocket formed by Tyr123, Phe125, and Tyr170. This 29 finding is further supported by the highly improved enantioselectivity of the mutant Est-Y29 (F125W) toward $(S)$-ketoprofen due to the enhanced $\pi$-interaction. Our results provide the molecular basis of the enantioselectivity of Est-Y29 against $(S)$-ketoprofen and further offer the opportunity for the rational design of enzyme enantioselectivity as well as potential applications of the mutant Est-Y29 to industrial biocatalysts.

35 Key words: crystal structure, esterase, Est-Y29, rational design, enantioselectivity, $(S)$ 36 ketoprofen 


\section{1. Introduction}

38 Biocatalysts have been under development for several decades to replace chemical-based synthesis because they have advantages in economic feasibility and environmental friendliness

1. In addition, their higher specificity in regio- and stereo-isomers is useful for reducing synthesizing steps, which is a key consideration for industrial applications of biocatalysts. Therefore, high stereoselectivity is one of the most important functional features for using enzymes as biocatalysts.

Many biochemical features of enzymes such as stability, activity, and selectivity must be considered for their various industrial applications from pharmaceuticals and green chemistry to biofuels ${ }^{2-3}$. To improve enzyme properties for industrial applications, two strategies have been widely used: directed evolution and rational design. Directed evolution, conducted by a combination of random mutagenesis and high-throughput screening, can be applied when structural information is not available and the assay system is well established 4-9. Conversely, rational design relies on the availability of structural information and the knowledge of catalytic mechanisms ${ }^{10-13}$. Although directed evolution has emerged as a popular tool in this field, it has limitations in that a huge number of random mutants, whose numbers exponentially increase in direct relation to the number of amino acids in the enzyme, must be screened to cover all possible combinations. Therefore, when the information of structures and enzyme mechanisms is available, rational design has proven to be an effective approach. In the rational design approach to acquire the enhanced enantioselectivity, the principle of mutation is to either stabilize the faster-reacting enantiomers or to destabilize the slower-reacting ones in the transition state by mutating the residues that affect interaction between the residues and any substituents at the chiral carbon of substrates ${ }^{10,13}$. cyclooxygenases (COXs), has been widely used as a non-steroidal anti-inflammatory drug 
(NSAID) ${ }^{14-15}$. There are two stereoisomers of ketoprofen with one chiral center: $(R)-(-)-$ and $(S)-(+)$-forms. In terms of inhibitory power to COXs, $(S)$-ketoprofen was shown to be 100 times stronger than $(R)$-ketoprofen ${ }^{16}$. Subsequent studies also revealed that the $S$-enantiomer had higher suppressive effects of $\mathrm{PGE}_{2}$ production in macrophages and to edema, whereas the $R$ enantiomer only showed effects as a pain killer ${ }^{17-18} .(R)$-ketoprofen has also been used as an additive in a toothpaste for the purpose of reducing periodontal disease ${ }^{19}$. Due to differences in physiological functions of ketoprofen isomers, the Food and Drug Administration (FDA) requested to separate each enantiomer for specific usage ${ }^{20-21}$. Ong et al. summarized several approaches for the effective production of the $(S)$-enantiomer of ketoprofen, including diastereomer crystallization, kinetic resolution, asymmetric synthesis, chromatography separation, and preferential crystallization ${ }^{22}$. Among them, kinetic resolution by enantiospecific esterification or hydrolysis has been most widely used because it has no unexpected side product and is an environment-friendly process with efficient processing steps.

Est-Y29, a family VIII lipolytic esterase isolated from metagenomes, isolated from the topsoil in the area of Chungcheong-bukdo, Republic of Korea (Microbial Genomics and Application Center, Republic of Korea), showed moderated enantioselectivity against $(S)$ ketoprofen ethyl ester (Scheme 1) ${ }^{23}$. This enzyme is a promising catalyst for producing the medicinally active enantiomer of ketoprofen due to its high stability in organic solvents, acidic and basic conditions, and high temperature conditions ${ }^{23}$. However, since the enantioselectivity of Est-Y29 was only marginal $(E$ value $=4.48)$, its enantioselectivity must be improved to use Est-Y29 as a biocatalyst for producing $(S)$-ketoprofen. Previously we solved the crystal structures of Est-Y29 in apo (PDB code: 4P6B) and inhibitor-bound (PDB code: 4P87) forms, which reveal that Est-Y29 belongs to the $\alpha / \beta$ hydrolase fold with S58-K61-Y170 catalytic triad 24-25. For the purpose of applying a rational design approach to improve the enantioselectivity of Est-Y29, we solved the crystal structures of Est-Y29 in complexes with (S)-ketoprofen. In 
87 addition to the structural interpretation of the complex structure, based on the docking studies of $(R)$ - and $(S)$-ketoprofens ethyl ester and structural analyses of Est-Y29 mutants in a complex with $(S)$-ketoprofen ethyl ester, we found the structural bases for the enantioselectivity of Est-

90 Y29. Furthermore, by this approach, we designed a mutant that has 5-fold higher 91 enantioselectivity than the wild-type Est-Y29. Therefore, this study not only provides the 92 molecular basis for the enantioselectivity of the ester hydrolysis enzyme but also produces a 93 potential biocatalyst with an industrial application for the synthesis of a medicinally active 94 enantiomer of ketoprofen.

95

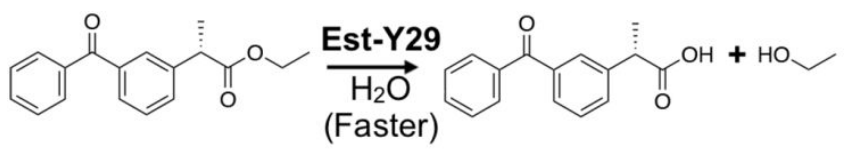

96

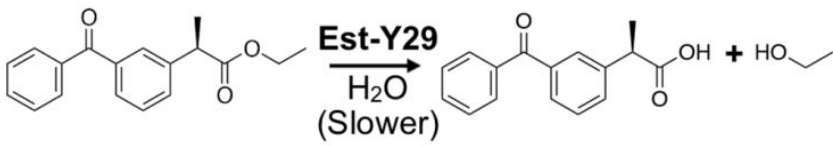

97 Scheme 1. Enantioselective Hydrolysis of Racemic $(R, S)$-Ketoprofen Ethyl Ester by

\section{Esterase Est-Y29}


100 2. Materials and Methods

101

102

103

104

105

106

107

108

109

110

111

112

113

114

115

116

117

118

119

120

121

122

123

\subsection{Preparation of Racemic and $(S)$-Ketoprofen Ethyl Ester}

Substrates for Est-Y29 and its mutants were prepared from commercially available $(S)$ ketoprofen (Sigma-Aldrich, MO, USA), $(R)$-ketoprofen (Toronto Research Chemicals, ON, Canada), and racemic ketoprofen (Tokyo Chemical Industry, Japan). As previous description for esterification ${ }^{26}, 0.2 \mathrm{mM}$ of racemic ketoprofen dissolved in $2 \mathrm{~mL}$ of ethanol was incubated at $333 \mathrm{~K}$ for $5 \mathrm{~h}$ with a $10 \mu \mathrm{L}$ of concentrated sulfuric acid as a catalyst. After removal of residual ethanol by heating in vacuo, the mixture was poured into saturated $\mathrm{NaHCO}_{3}$, and the aqueous layer was extracted with ethyl acetate. The combined organic phases were washed with brine, dried over $\mathrm{Na}_{2} \mathrm{SO}_{4}$, filtrated, and concentrated under reduced pressure. The residue was purified by flash chromatography on silica gel using 1:3 mixture of ethyl acetate and hexanes to give racemic ketoprofen ethyl ester as light yellowish liquid. The structure of racemic ketoprofen ethyl ester was confirmed by ${ }^{1} \mathrm{H}$ and ${ }^{13} \mathrm{C}$ NMR spectroscopy (Figure $\mathrm{S} 1$ ). $(S)$-ketoprofen ethyl ester and $(R)$-ketoprofen ethyl ester were also prepared in the same procedure.

\subsection{Protein Purification and Crystallization}

Protein expression and purification were performed as described elsewhere with slight modifications ${ }^{24}$. Briefly, the pQE30-Est-Y29 construct was transformed into Escherichia coli XL1-Blue cells (Agilent, CA, USA) inoculated in $500 \mathrm{~mL}$ of LB medium containing 100 $\mu \mathrm{g} \cdot \mathrm{mL}^{-1}$ ampicillin. Protein induction was performed at $310 \mathrm{~K}$ for $4 \mathrm{~h}$ using $1 \mathrm{mM}$ isopropyl$\beta$-D-1-thiogalactoside (IPTG) as final concentration. Cells were harvested through centrifugation at $6,000 \mathrm{~g}$ for $15 \mathrm{~min}$, suspended in buffer A $(50 \mathrm{mM}$ Tris- $\mathrm{HCl}, 300 \mathrm{mM} \mathrm{NaCl}$ with $50 \mathrm{mM}$ imidazole a $\mathrm{pH} 8.0$ ), and disrupted by sonication. After centrifugation at $69,000 \mathrm{~g}$ 
124 for $30 \mathrm{~min}$, the supernatant was applied to a Ni-NTA column (GE Healthcare, IL, USA) 125 equilibrated with the buffer A, followed by an extensive washing step using buffer A to remove non-specifically bound proteins. Subsequently, the bound protein was eluted using 150-450 $\mathrm{mM}$ of imidazole-gradient buffer A, and from the collected protein, homogenous Est-Y29 was obtained using HiLoad 16/600 Superdex 200 column (GE Healthcare, IL, USA) with $10 \mathrm{mM}$ phosphate buffer $\mathrm{pH} 8.0$. The finally purified Est-Y29 was concentrated to $10 \mathrm{mg} \cdot \mathrm{mL}^{-1}$ with buffer change using an Amicon Ultra-15 centrifugal filter, 10 kDa MWCO (Merk Millipore, Germany), and stored at $193 \mathrm{~K}$ until the next use. Purification of Est-Y29 mutants (F125W, R225A and A348V) was performed in the same way as that of native protein.

Crystallization was performed using the microbatch (Thermo Fisher Scientific, Denmark) crystallization method under a thin layer of Al's oil, using commercially available screening kits from Hampton Research (Hampton Research, CA, USA) at 295 K. Est-Y29 was mixed with $(S)$-ketoprofen ethyl ester to a final concentration of $8 \mathrm{mg} \cdot \mathrm{mL}^{-1}$ for protein and 1 $\mathrm{mM}$ for $(S)$-ketoprofen ethyl ester and incubated $5 \mathrm{~min}$ before crystal setting. Diffractionquality crystals of Est-Y29 F125W and a complex of Est-Y29 with (S)-ketoprofen (Est-Y29SKP) were obtained from the reservoir containing $1 \mathrm{M}$ sodium citrate and $100 \mathrm{mM}$ sodium acetate $\mathrm{pH} 4.6$ after one day incubation at $287 \mathrm{~K}$. We used the same approach to crystallize complexes of Est-Y29 A348V with (S)-ketoprofen ethyl ester (Est-Y29 A348V-SKE) with EstY29-SKP except we increased the incubation time to $30 \mathrm{~min}$.

\subsection{X-ray Data Collection}

Crystals of the complex of Est-Y29-SKP, Est-Y29 F125W and Est-Y29 A348V-SKE were transferred to a cryosolution containing $1 \mathrm{M}$ sodium citrate, $100 \mathrm{mM}$ sodium acetate $\mathrm{pH} 4.6$, $25 \%$ glycerol, and $1 \mathrm{mM}(S)$-ketoprofen ethyl ester. X-ray diffraction data were collected at $100 \mathrm{~K}$ using an ADSC Quantum 210 detector on beamline NW12 at Photon Factory (PF) in 
149 Japan and an ADSC Quantum 315 CCD on beamline PAL 7A at the Pohang Accelerator 150 Laboratory (PAL) in Korea. The data were indexed, integrated, and scaled using HKL-2000 27.

151 Data collection and processing statistics for Est-Y29-SKP, Est-Y29 F125W, and Est-Y29 152 A348V-SKE are summarized in Table 1.

153

$154 \quad$ 2.4 Structure Determination and Refinement

155 The structure of apo-Est-Y29 (PDB code: 4P6B) was used as a template for molecular 156 replacement to solve the structure of Est-Y29-SKP, Est-Y29 F125W and Est-Y29 A348V-SKE 157 using MOLREP ${ }^{28}$. The model was refined using REFMAC ${ }^{29-30}$ and PHENIX ${ }^{31}$. The quality 158 of the final model was evaluated with the program PROCHECK ${ }^{32}$. Refinement statistics are 159 given in Table 1. The structure models were described using PyMOL, and interactions between 160 protein and ligand were visualized using BIOVIA Discovery Studio (Accelrys, CA, USA). The 161 final models Est-Y29-SKP (PDB code: 5ZWR), Est-Y29 F125W (PDB code: 5ZWV) and Est162 Y29 A348V-SKE (PDB code: 5ZWQ) were deposited in PDB.

164 Table 1. Data Collection and Refinement of the Complex Structures of Est-Y29-SKP and 165

\section{Mutants, Est-Y29 F125W and Est-Y29 A348V-SKE .}

$\begin{array}{ccc}\text { Est-Y29-SKP } & \text { Est-Y29 F125W } & \text { Est-Y29 A348V-SKE } \\ (5 Z W R) & (5 Z W V) & (5 Z W Q)\end{array}$

\section{Data Collection}

Space Group

I4

I4

I4

\section{Cell Dimensions}
$a, b, c(\AA)$
$122.3,122.3,155.8$
$121.8,121.8,155.4$
$122.3,122.3,155.2$
$\alpha, \beta, \gamma\left(^{\circ}\right)$
$90.0,90.0,90.0$
$90.0,90.0,90.0$
$90.0,90.0,90.0$

Molecules/AU

2

2

2 


\begin{tabular}{|c|c|c|c|}
\hline Wavelength & 1.0000 & 1.0000 & 1.0000 \\
\hline Resolution $(\AA)$ & $50-1.69$ & $50-2.10$ & $50-1.80$ \\
\hline$R_{\text {sym }}$ or $R_{\text {merge }}{ }^{\mathrm{b}}$ & $5.2(29.2)$ & $8.0(31.7)$ & $8.0(37.8)$ \\
\hline Mean $I / \sigma(I)$ & $49.9(8.3)$ & $34.5(8.1)$ & $24.7(3.4)$ \\
\hline Completeness (\%) & $99.9(99.9)$ & $99.9(99.7)$ & $99.7(98.4)$ \\
\hline Redundancy & $7.4(7.3)$ & $7.5(7.5)$ & $3.7(3.7)$ \\
\hline \multicolumn{4}{|l|}{ Refinement } \\
\hline Resolution $(\AA)$ & $50-1.69$ & $50-2.10$ & $50-1.80$ \\
\hline No. Reflections & 127264 & 65885 & 105315 \\
\hline$R_{\text {work }}{ }^{\mathrm{c}} / R_{\text {free }}{ }^{\mathrm{d}}$ & $15.57 / 17.78$ & $15.01 / 19.01$ & $16.22 / 18.87$ \\
\hline \multicolumn{4}{|l|}{ No. Atoms } \\
\hline Protein & 6122 & 6040 & 5996 \\
\hline Ligand/Ion & 50 & - & 54 \\
\hline Water & 1124 & 797 & 827 \\
\hline \multicolumn{4}{|l|}{$B$-factors } \\
\hline Protein & 16.54 & 21.80 & 21.04 \\
\hline Ligand/Ion & 26.07 & - & 42.59 \\
\hline Clash Score & 3.69 & 2.09 & 2.01 \\
\hline \multicolumn{4}{|l|}{ R.M.S.D. ${ }^{\mathrm{e}}$} \\
\hline Bond Lengths $(\AA)$ & 0.007 & 0.007 & 0.012 \\
\hline Bond Angles $\left({ }^{\circ}\right)$ & 1.047 & 0.833 & 1.159 \\
\hline \multicolumn{4}{|l|}{ Ramachandran (\%) } \\
\hline Favored & 97.6 & 96.77 & 97.01 \\
\hline
\end{tabular}




$\begin{array}{llll}\text { Allowed } & 2.33 & 3.23 & 2.99 \\ \text { Outliers } & 0.00 & 0.00 & 0.00\end{array}$

166

167 a Values in parentheses refer to the last resolution shell.

$168{ }^{\mathrm{b}} R_{\text {symm }}=\sum_{\mathrm{h}} \sum_{\mathrm{i}}\left|I\left(h_{i}\right)-<I(h)>\right| / \sum_{\mathrm{h}} \sum_{\mathrm{i}} I\left(h_{i}\right)$, where $I\left(h_{i}\right)$ is the single intensity of reflection $h$ as

169 determined by the $i$ th measurement and $\langle I(h)>$ is the mean intensity of reflections $h$.

$170{ }^{\mathrm{c}} R_{\text {work }}(\%)=\sum\left|F_{O}-F_{C}\right| / \sum F_{O}$, where, $F_{O}$ is the observed structure factor amplitude and $F_{C}$

171 is the structure factor calculated from the model.

$172{ }^{\mathrm{d}} R_{\text {free }}(\%)$ is calculated in the same manner as $R_{\text {work }}$ using $5 \%$ of all reflections excluded from

173 refinement stages using high resolution data.

174 eR.M.S.D., root-mean-square deviation.

\subsection{Structure Analysis}

178 The distances between two interacting atoms or center of aromatic rings were calculated using the Discovery Studio (Accelrys, CA, USA). Hydrogen bonds, $\pi$-interactions $(\pi-\sigma, \pi-\pi$, and $\pi$ alkyl), salt-bridges were identified based on the type of interaction and their distances in the

Discovery Studio. The default values were used. Cavity volumes of the wild-type, and mutant EST-Y29 were calculated using CASTp3.0 ${ }^{33}$ after removing the bound ligands in the case of the wild-type enzyme. For the calculation of the cavity volume of the F125W mutant, F125 in the wild-type structure was mutated to tryptophan residue followed by energy minimization since residues 225-227 in the flexible loop occupy the active site cavity.

\subsection{Docking Simulation}


188 The crystal structure of Est-Y29-SKP was used as a docking template for the docking to the

wild-type and F125W EST-Y29. The crystal structure of the A348V mutant bound to SKE was

used for the docking template for the docking to the A348V mutant. All resolved water molecules were removed from the structure prior to protein preparation and subsequent docking simulations. Template structures were minimized using L-BFGS-B with GalaxyRefine $^{34}$ energy before docking was performed. The F125W mutant structure was prepared by mutating F125 of wild type enzyme to tryptophan residue followed by local minimization using L-BFGS-B.

AutoDock Tool ${ }^{35}$ was used to create a .pdbqt file, which is a modified PDB file containing 1) the coordinates of protein and substrates, $(S)$-ketoprofen ethyl ester and $(R)$ ketoprofen ethyl ester, and 2) additional information such as partial charges, atom type, and topology. Substrate docking to the Est-Y29 was performed using AutoDock Vina ${ }^{36}$ with W125, R225, and L227 defined as flexible side chains. Subsequently, the docking space was visually defined in AutoDock Tool. The grid box around the catalytic triads with a dimension of $20 \AA \mathrm{x}$ $32 \AA \times 20 \AA$ was used to cover the entire substrate-binding site and to accommodate ligands. Defaults parameters were used during the ligand docking except exhaustiveness, which was adjusted to 1000. Calculations for ligand docking were done using the Lamarckian Genetic Algorithm (LGA) method ${ }^{37}$. Among the top 10 candidates, the final model was chosen based on interaction geometry with the catalytic triad.

To consider additional side-chain conformation changes and subtle backbone movements upon binding, the complex structure generated by Autodock Vina was further refined using GalaxyRefine ${ }^{34,38}$. Among 24 models generated by GalaxyRefine, the model having best GalaxyDock BP2 Score ${ }^{39}$ was selected as the final model.

\subsection{Site-directed Mutagenesis}


213 Site-directed mutagenesis was performed using the QuickChange kit (Stratagene, CA, USA),

214 and results were confirmed by DNA sequencing. The introduction of single site mutations

215 into Est-Y29 was achieved by the use of pQE30-Est-Y29 as a template together with the primer

216 sets described in Table 2. Bold nucleic acids with underlines in primer sequences indicate the

217 codon or anticodon of a mutant. Mutagenesis at each site was performed using polymerase

218 chain reaction (PCR). Briefly, after initial activation of $P f u$ polymerase (Cosmo Genetech,

219 Korea) at $368 \mathrm{~K}$ for $5 \mathrm{~min}, 15$ repeats of the cycle including denaturation at $368 \mathrm{~K}$ for $30 \mathrm{~s}$,

220 annealing at $328 \mathrm{~K}$ for $30 \mathrm{~s}$, and extension at $345 \mathrm{~K}$ for $12 \mathrm{~min}$, followed. Then, an extra

221 extension was given at $345 \mathrm{~K}$ for $15 \mathrm{~min}$, and the product was stored at $277 \mathrm{~K}$. To separate the

222 parental template from the mutated template, the product was incubated with 10 unit of $D p n I$

223 (New England Biolabs, MA, USA) at $310 \mathrm{~K}$ for $1 \mathrm{~h}$ before being transformed into competent

224 E. coli XL1-Blue cells. Colonies positive to $50 \mu \mathrm{g} \cdot \mathrm{mL}^{-1}$ ampicillin were selected, and

225 sequences of extracted plasmid were identified.

226

227

\section{Table 2. Primer Information to Generate Est-Y29 Mutants}

\begin{tabular}{|c|c|}
\hline Mutation & Sequence \\
\hline \multicolumn{2}{|l|}{ F125W } \\
\hline Forward & $5^{\prime}-\mathrm{G}$ GCT GGC TTT TCA TAT GAT TGG TCG TTG GGA TGC CC-3' \\
\hline Reverse & $5^{\prime}-\mathrm{GG}$ GCA TCC CAA CGA CCA ATC ATA TGA AAA GCC AGC C-3' \\
\hline \multicolumn{2}{|r|}{ 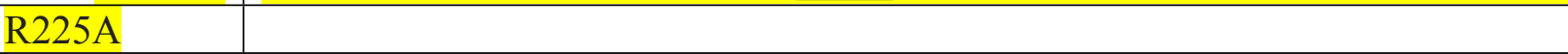 } \\
\hline Forward & $5^{\prime}-$ GAA GTG TAC GGA ATG GCG TCT CTT CAC GGA CTG-3' \\
\hline Reverse & $5^{\prime}-\mathrm{CAG}$ TCC GTG AAG AGA CGC CAT TCC GTA CAC TTC-3' \\
\hline \multicolumn{2}{|r|}{ 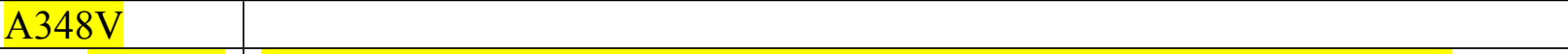 } \\
\hline Forward & $5^{\prime}$-TTC GGT TGG AGC GGA GTC GCA GCA ACA TAT TTT-3' \\
\hline Reverse & 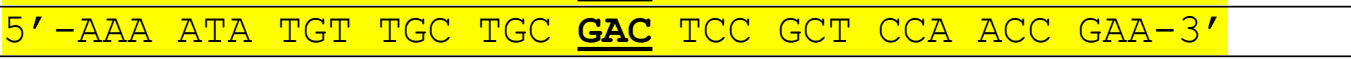 \\
\hline
\end{tabular}

228

229

\subsection{Thermal stability assay}

230 Thermo-stabilities of the wild-type and F125W Est-Y29 were tested using circular dichroism

231 (CD) spectroscopy (Jasco, Easton, MD, USA). Both the wild-type and mutant Est-Y29 were

prepared as previously described at the concentration of $50 \mu \mathrm{M}$ in the buffer containing $20 \mathrm{mM}$ 
233 phosphate $\mathrm{pH} 8.0$. Wavelength was scanned from $190 \mathrm{~nm}$ to $250 \mathrm{~nm}$ at $297 \mathrm{~K}$, and the optimal 234 point was selected at $217 \mathrm{~nm}$, which showed the absolute maximum value in helical structure.

235 Thermostability was tested at $217 \mathrm{~nm}$ by increasing the temperature from $283 \mathrm{~K}$ to $363 \mathrm{~K}$.

236 Results were analyzed by three-segment linear equation, and the melting temperature $\left(\mathrm{T}_{\mathrm{m}}\right)$ was

237 calculated by average value of interaction points between two etquations. Each sample was 238 measured in triplicate and statistically analyzed using Student's T-test. To measure the stability 239 of the wild-type and mutant Est-Y29 in the presence of substrates, Est-Y29 at the concentration 240 of $50 \mu \mathrm{M}$ was mixed with $400 \mu \mathrm{M}$ of $(S)$-ketoprofen ethyl ester or $(R)$-ketoprofen ethyl ester, 241 and their $T_{m}$ values were determined by monitoring the ellipticity change at $246 \mathrm{~nm}$ in the temperature ranges from $323 \mathrm{~K}$ to $363 \mathrm{~K}$. Due to noise at the high energy wavelength possibly caused by the presence of DMSO in the buffer, ellipticity change was monitored at $246 \mathrm{~nm}$.

\subsection{Activity Assays}

246 To test functional thermostability, Est-Y29 were prepared after heat exposure. Est-Y29 was 247 incubated in various temperature points from $308 \mathrm{~K}$ to $363 \mathrm{~K}$ for $30 \mathrm{~min}$, and was cooled down 248 at $298 \mathrm{~K}$ for $10 \mathrm{~min}$. For analysis of residual hydrolysis activity, initial velocity of hydrolysis of $p$-nitrophenol butyrate ( $p$-NB) was measured by chasing color change at $405 \mathrm{~nm}$ wavelength 250 time-dependently. Residual enantioselective catalytic activity of Est-Y29 was also verified using $(R)$ - and $(S)$-Roche ester (methyl 3-hydroxy-2-methylpropionate) as substrates through color change of $\mathrm{pH}$ indicator, phenol red, and color change was measured at $430 \mathrm{~nm}$ wavelength time-dependently. Each reaction was repeated 3-4 times, and its initial velocities were averaged.

Enzyme reactions were preceded with $1 \mathrm{mM}$ racemic ketoprofen ethyl ester in $10 \mathrm{mM}$

256 Tris- $\mathrm{HCl}$ buffer $\mathrm{pH} 8.0$ at $323 \mathrm{~K}$. Conversion ratio of racemic ketoprofen to initial racemic ketoprofen ethyl ester was measured using NMR. The products, $(R)$ - and $(S)$-ketoprofen, were 
analyzed using a chiral compound analytical column, Chirex Phase 3005 (Phenomenex Co., CA, USA) mounted on HPLC (Agilent, CA, USA) and UV detector at $254 \mathrm{~nm}$. The mobile phase contained $0.03 \mathrm{M}$ ammonium acetate and $70 \%$ methanol. To obtain appropriate $E$ value, conversion ratio was controlled to be less than 0.5. Enantiomeric excess of product $\left(e e_{P}\right)$, conversion (c), and $E$ value were calculated using the following equations:

$E=\ln \left[1-c\left(1+e e_{P}\right)\right] / \ln \left[1-c\left(1-e e_{P}\right)\right]$

where $C_{S}$ and $C_{R}$ represent concentrations of $(S)$-ketoprofen and $(R)$-ketoprofen, respectively, in the hydrolyzed products, and $C_{e 0}$ represents the initial concentration of substrate racemic ketoprofen ethyl ester in the hydrolysis reaction.

\subsection{Enzyme Kinetic Analysis}

Enzyme kinetic parameters of the wild-type and F125W Est-Y29 were obtained by measuring the rate of hydrolysis of either the pure $(R)$ - or $(S)$-ketoprofen ethyl esters at various substrate concentrations at $323 \mathrm{~K}$ for $5-10 \mathrm{~min}$, which was in the range of initial rates, in $50 \mathrm{mM}$ Tris$\mathrm{HCl}$ buffer $(\mathrm{pH} 8.0)$. The enzyme concentration was $0.3 \mathrm{mg} \cdot \mathrm{mL}^{-1}$. The reaction rate was determined by comparing the amount of product with the residual reactant at the time of stopping reaction using high performance liquid chromatography (HPLC). Agilent 1260 Infinity HPLC system was used equipped with the column Chiralpak IC (4.6 mm x $250 \mathrm{~mm}$ ). The eluent was $10 \%$ IPA and hexane ( $0.1 \%$ Trifluoroacetic acid), and the area of the peak observed under UV (254 nm) wavelength was used to determine the reaction rate. The enzyme kinetic parameters, $\mathrm{K}_{\mathrm{m}}$ and $\mathrm{V}_{\max }$ were determined using GraFit 7 (Erithacus Software, Surrey, 281 UK).

\section{3. Results}




\section{3 \\ 3.1 Structural Studies of Est-Y29 in Complex with (S)-Ketoprofen}

284 To explain the binding mode and selectivity of Est-Y29 for the $S$-enantiomer, we attempted to

identify the complex structure of Est-Y29 and $(S)$-ketoprofen ethyl ester by co-crystallizing the protein and the substrate under low $\mathrm{pH}$ conditions ( $\mathrm{pH} 4$ 4.6). However, upon crystallization, we determined that we obtained the (S)-ketoprofen-bound structure of Est-Y29, named Est-Y29SKP, instead of the (S)-ketoprofen ethyl ester-binding form (Figure 1A). Yoon et al. characterized the activity of Est-Y29 as below $20 \%$ under acidic conditions ${ }^{23}$, and thus it is assumed that hydrolysis of (S)-ketoprofen ethyl ester occurs during incubation and crystallization. Although Est-Y29-SKP fails to directly reveal the ethyl substituent in the ester bond of $(S)$-ketoprofen ethyl ester, it still provides insight into the enzyme-substrate interactions through benzophenone and the chiral center of the $S$-enantiomer in the product complex.

The overall structure of the complex Est-Y29-SKP is very similar to the apo Est-Y29 structure (PDB 4P6B) with a root mean square deviation (RMSD) value of $0.13 \AA$ for 2573 atoms out of all 2943 atoms in the Est-Y29 (residue 1-389) (Figure 1A). Atoms in the loop containing residues 225-230 were not included for RMSD calculation. In the apo-structure, the electron density map at the loop 225-230 in Est-Y29 is poorly defined, which suggests that this loop is more flexible in the absence of ligand. In the Est-Y29-SKP structure, two residues in this loop, R225 and L227, interact directly with the phenol group of the (S)-ketoprofen (Figure 1A). The substrate position in the binding pocket can be stabilized due to the movement and interaction of the flexible loop 225-230 with the substrate. Thus, the volume of the pocket in 304 Est-Y29 shows a difference between the apo and (S)-ketoprofen-binding forms (Figure 1B).

305 The electron density map $2 F_{O^{-}} F_{C}$ of $(S)$-ketoprofen is clearly defined in the active site of Est306 Y29-SKP (Figure 1C). This suggests that $(S)$-ketoprofen locates in a substrate-binding pocket 307 of Est-Y29 with high occupancy. (S)-ketoprofen is stabilized in the binding site of Est-Y29 by 
308 numerous favorable interactions. In detail, binding of $(S)$-ketoprofen occurs by direct hydrogen

309 bonds of $(S)$-ketoprofen with main-chain S58 (3.1 $\AA)$ and A348 (3.0 $\AA)$, which form the

310 oxyanion hole and the catalytic S58 (Figure 1D). Furthermore, the oxygen in the benzophenone

311 group makes a water-mediated hydrogen bond with residue Y57 and S248, and many $\pi$

312 interactions help to hold the benzophenone of (S)-ketoprofen. Specifically, residues R225,

313 L227, and L370 stabilize one phenyl group of (S)-ketoprofen, while residues Y57, I141, H261,

314 and A348 interact with the other phenyl group via various $\pi-\sigma, \pi$-cation, $\pi-\pi$, and $\pi$-alkyl

315 interactions (Figure 1E). Importantly, the methyl substituent at the chiral carbon center makes

316 three $\pi$-alkyl interactions with residues Y123, F125, and Y170 with the distances of 4.4, 4.6

317 and $5.1 \AA$, respectively. These aromatic interactions explain why Est-Y29 has a preference for

318 the $(S)$-ketoprofen ethyl ester.

319 

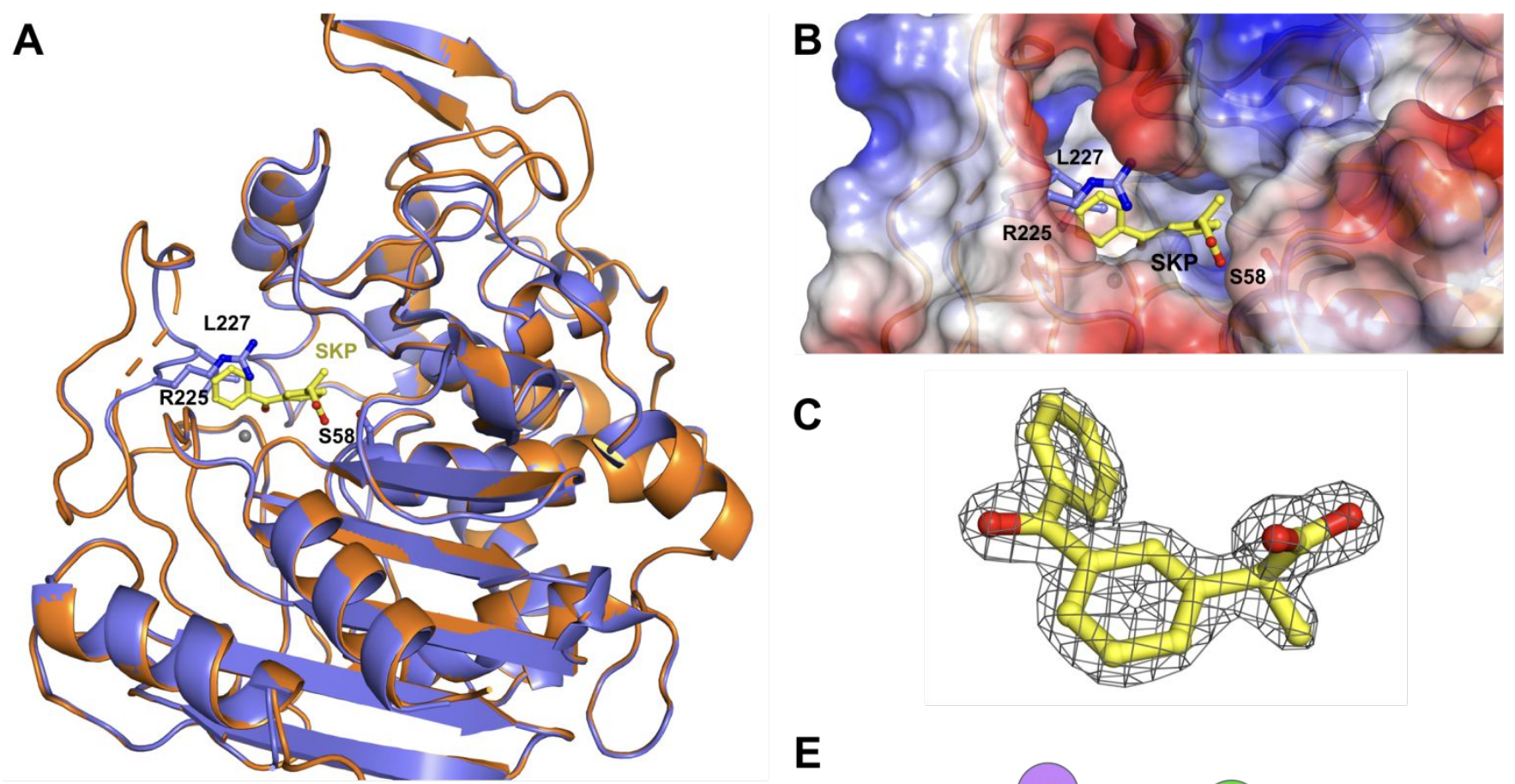

$\mathbf{E}$
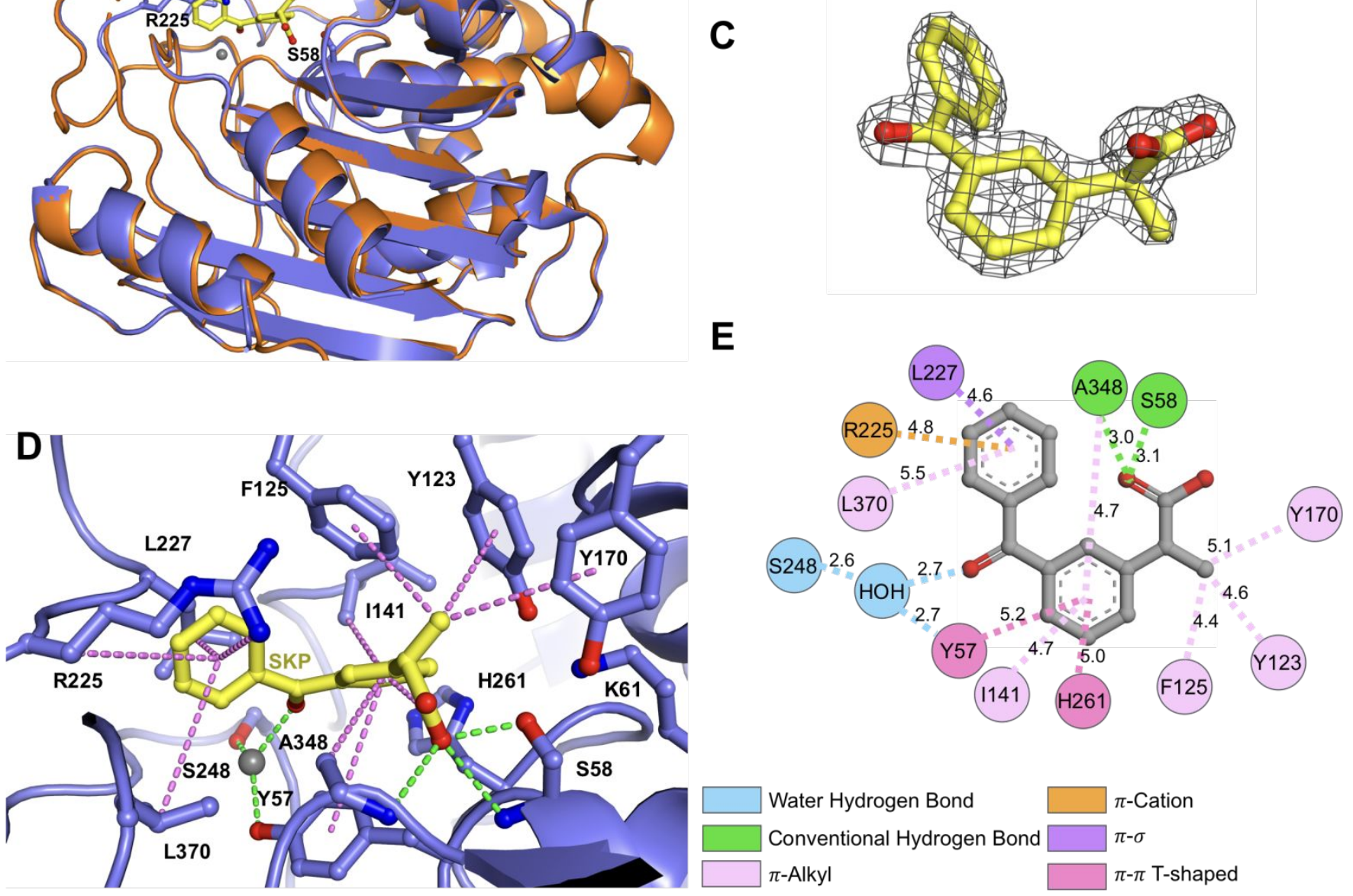

Figure 1. Complex Structure of the Est-Y29 with (S)-Ketoprofen. (A) Superimposition of

323 the apo Est-Y29 (orange cartoon representation, PDB ID: 4P6B) and the complex Est-Y29 with

$324(S)$-ketoprofen (slate cartoon representation). (S)-ketoprofen is shown in yellow in the stick 325 representation. Specific residues on the flexible loop 225-230 are shown in the slate stick 326 representation. The dashed tube represents the missing residues in the apo Est-Y29 structure. 327 (S)-ketoprofen is marked as SKP in the figure. (B) Surface representation colored by the 328 vacuum electrostatic potential of apo Est-Y29 in the same orientation as in (A). (C) $2 \mathrm{~F}_{\mathrm{O}}-\mathrm{F}_{\mathrm{C}}$ 329 map contoured at $1.5 \sigma$ shows clear electron density for $(S)$-ketoprofen, shown in yellow stick 330 representation. (D) Detailed interactions of $(S)$-ketoprofen (yellow sticks) and residues in the 331 active site of Est-Y29 (slate stick representation). The water molecule involved in substrate 
332

333

334

335

336

337

338

339

340

341

342

343

344

345

346

347

348

349

350

351

352

353

354

355

356

357

358

binding is shown as grey sphere. Hydrogen bonds are indicated with green dashed lines. $\pi$ interactions ( $\pi$-cation, $\pi-\sigma, \pi-\pi$, and $\pi$-alkyl) are shown with violet dashed lines. S58 is the catalytic residue. An oxyanion hole is formed by the main chain NH of S58 and A348. (E) 2D ligand interaction diagram of $(S)$-ketoprofen with the Est-Y29 active site. Atomic interactions are indicated by the dotted lines and their distances in angstroms are shown.

\subsection{Docking Simulation of Est-Y29 with $(R)$ - and $(S)$-Ketoprofen Ethyl Ester}

To get insight into the enantioselectivity of Est-Y29, we performed induced-fit docking studies of each substrate, $(S)$ - and $(R)$-ketoprofen ethyl esters, separately. We used the crystal structures of Est-Y29-SKP a template for the docking study after removing $(S)$-ketoprofen and glycerol. For the induced-fit docking analysis, key residues involved in ligand binding were allowed for flexible movement during docking. Then, the docking models were further refined using GalaxyRefine ${ }^{34,}{ }^{38}$, which is used for iterative sidechain repacking and the relaxation of the overall structures. The induced-fit docking approach used in this study was successfully validated in the previous study of the GPCR-ligand docking ${ }^{40}$.

The induced-fit docking models show that $(S)$-ketoprofen ethyl ester binds to the pocket occupied by $(S)$-ketoprofen in the crystal structure of Est-Y29-SKP, suggesting that the docking was successful. In addition, the docking model was validated by that the carbonyl oxygen of the $(S)$-enantiomer makes hydrogen bonds with oxyanion hole formed by the NH of S58 (3.4 A) and A348 (2.9 A), (Figure 2A). Residues L227, R225, L370, and A348 stabilize the benzophenone group by $\pi$-alkyl interactions (Figure 2B). F125 and Y123 also stabilize benzophenone group by $\pi-\pi$ interactions. The methyl substituent at the chiral center makes a $\pi$-alkyl interaction with residues Y123, F125, and Y170 at distances of $5.5 \AA, 4.3 \AA$ and $5.2 \AA$, respectively (Figure 2A). When the active site of the docking model of Est-Y29-SKE is superimposed over the crystal structure Est-Y29-SKP, two ligands $((S)$-ketoprofen and $(S)$ ketoprofen ethyl ester) occupy the same position, and the key interactions in both structures are well conserved (Figure S2A), thus verifying the accuracy of the docking model of Est-Y29- 
359 SKE. The docking model of Est-Y29 with $(R)$-ketoprofen ethyl ester (Est-Y29-RKE) also binds

360 Est-Y29 in a productive mode (Figure 2C), in which the carbonyl oxygen forms hydrogen

361 bonds with S58 (3.4 $\AA$ ) and A348 (2.8 $\AA$ ). Key residues stabilizing the benzophenone group of

362 (R)-ketoprofen ethyl ester by $\pi$-interactions include I141, L227, L370, H261, and A348 (Figure

363 2D). The residues stabilizing the benzophenone and carbonyl oxygen of the ligands are highly

364 conserved between the two docking structures. Superimposing the docking model Est-Y29-

365 RKE onto the crystal structure of Est-Y29-SKP suggests that the binding mode of the docking

$366(R)$-ketoprofen ethyl ester and $(S)$-ketoprofen in the active site of Est-Y29 are similar (Figure

367 S2B). The most pronounced difference between the RKE- and SKE-bound docking models lies

368 on the opposite orientation of $\mathrm{CH}_{3}$ and $\mathrm{H}$ substituents in the chiral center (Figure $\mathrm{S} 2 \mathrm{C}$ ). Instead

369 of interacting with the aromatic pocket formed by residues Y123, F125, and Y170, the methyl

370 substituent of $(R)$-ketoprofen ethyl ester forms $\pi$-alkyl and alkyl interactions with the nearby

371 residues A348 and F125 (Figure 2C).

372 


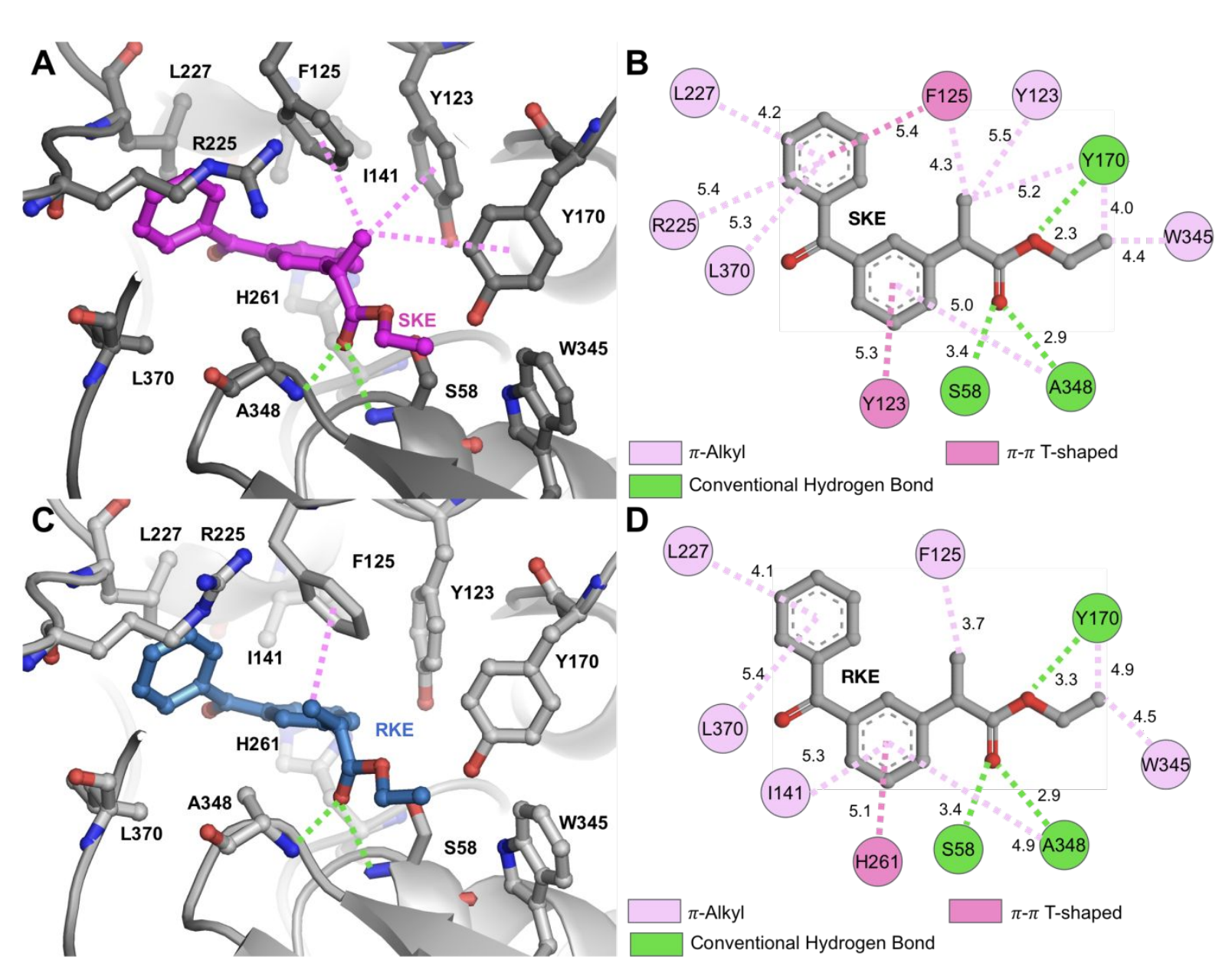

\section{Figure 2. Docking Models of $(R)$ - and $(S)$-Ketoprofen Ethyl Ester to Est-Y29}

(A) Detailed interactions of the docking (S)-ketoprofen ethyl ester (magenta sticks) and residues in the active site of Est-Y29 (grey stick representation). Hydrogen bonds are indicated with green dashed lines. $\pi$-interactions ( $\pi$ - $\pi$, and $\pi$-alkyl) are shown with violet dashed lines. (S)-ketoprofen ethyl ester is marked as SKE in the figure. (B) 2D ligand interaction diagram of docking $(S)$-ketoprofen ethyl ester with the Est-Y29 active site. (C) Detailed interactions of the docking $(R)$-ketoprofen ethyl ester (marine sticks) and residues in the active site of Est-Y29 (grey stick representation). Hydrogen bonds are indicated with green dashed lines. $\pi$-interaction is shown with violet dashed lines. $(R)$-ketoprofen ethyl ester is marked as RKE in the figure. (D) 2D ligand interaction diagram of docking $(R)$-ketoprofen ethyl ester with the Est-Y29 active site. In Figures (B) and (D), atomic interactions are indicated by the dotted lines and their distances in angstroms are shown. 


\subsection{Structure-based Design of Est-Y29 Mutants}

The crystal structure of the complex Est-Y29-SKP together with docking results

suggest that binding of two substituents $\mathrm{CH}_{3}$ and $\mathrm{H}$ at the chiral center of $(S)$-ketoprofen ethyl ester plays a major role determining Est-Y29 enantioselectivity; therefore, residues far away from these two substituents are likely not good targets for mutagenesis. To test this hypothesis, we designed a mutation at residue $\mathrm{R} 255 \mathrm{~A}$ to abolish the $\pi$-cation interaction between the R255 to the phenyl group of $(S)$-ketoprofen ethyl ester. Although residue R255 interacts directly with the ligand, it does not contribute to the binding of these two substituents. As we expected, mutation R255 did not affect the enantioselectivity since R255A showed a similar $E$ value to the wild-type enzyme (Table 3).

Since the aromatic pocket interacts directly with the $\mathrm{CH}_{3}$ substituent of $(S)$-ketoprofen

ethyl ester, it is expected that binding affinity of the $(S)$-enantiomer to protein can be enhanced by increasing $\pi$-interaction. As we expected, when replacing F125 to $\mathrm{W}$, the $E$ value increased from 4.48 to 20.39 (Table 3). Together with the approach to increase the binding of the $(S)$ enantiomer, we designed another mutant that has reduced binding affinity to $(R)$-enantiomer. The distance from chiral center in substrate to side chain $\mathrm{C} \beta$ in A348 is $3.7 \AA$, and the distance between $\mathrm{C} \alpha$ in $\mathrm{A} 348$ and $\mathrm{CH}_{3}$ substituent in $(S)$-substrate is $4.4 \AA$. The docking models revealed that $\mathrm{C} \beta$ atom in $\mathrm{A} 348$ is closer to the $\mathrm{CH}_{3}$ substituent of $(R)$-ketoprofen ethyl $(3.7 \AA)$ than that of $(S)$-ketoprofen ethyl ester (5.1 $\AA$ ) (Figures 2 and S2). Accordingly, it is expected that replacing A348 with a bulkier side chain residue would increase the repulsion force to the $(R)$-ketoprofen ethyl ester substrate, which makes the binding preference to $(R)$-ketoprofen ethyl ester smaller. Therefore, we expected that a mutation at A348 would lead to higher enantioselectivity by preventing the binding of the $R$-enantiomer to the active site of Est-Y29. 
413 Table 3. Enantioselectivity Values of Est-Y29 and Its Mutants toward (S)-Ketoprofena.

\begin{tabular}{|c|c|c|c|}
\hline protein & conversion & $\boldsymbol{e} \boldsymbol{e}_{\boldsymbol{P}}$ & $\boldsymbol{E}$ value \\
\hline Est-Y29 & 0.4045 & 0.5245 & 4.48 \\
\hline $\begin{array}{c}\text { Est-Y29 } \\
\text { R225A }\end{array}$ & 0.5339 & 0.4823 & 4.84 \\
\hline $\begin{array}{c}\text { Est-Y29 } \\
\text { A348V }\end{array}$ & 0.4686 & 0.3760 & 1.11 \\
\hline $\begin{array}{c}\text { Est-Y29 } \\
\text { F125W }\end{array}$ & 0.3598 & 0.8540 & 20.39 \\
\hline
\end{tabular}

$414{ }^{a}$ Representative HPLC analysis results are described in Figure S3.

$415 \quad 3.4$ Crystal Structure of A348V-SKE

416 To explain the poor enantioselectivity of mutant Est-Y29 (A348V) toward (S)-substrate

417 selectivity, we solved the crystal structure of the mutant A348V with its substrate $(S)$ -

418 ketoprofen ethyl ester using a co-crystallization approach. In this structure, we observed the

419 clear electron density of the ester substrate but not the hydrolyzed product (Figure 3A). Unlike

420 expectation from docking models, the longer and bulkier A348V residue was not stretched out

421 to the chiral center of the substrate, and eventually failed to bring direct hindrance in the space

422 for the methyl substituent in the chiral center of the $(R)$-ketoprofen ethyl ester. Rather, there

423 were less favorable interactions of substrate $(S)$-ketoprofen ethyl ester with the mutant Est-Y29

424 A348V. Two phenyl groups of the $(S)$-ketoprofen ethyl ester were stabilized by $\pi$-interactions

425 with residues Y57, I141, F125, H261, and V348. The oxygen of the benzophenone group

426 makes a water-mediated hydrogen bond with residue Y57 and S248, but there is no hydrogen

427 bond between (S)-ketoprofen ethyl ester and the oxyanion hole (V348 and S58) as was

428 observed in the Est-Y29-SKP crystal structure (Figures 3B and C). When compared to $(S)$ -

429 ketoprofen bound to Est-Y29, the $(S)$-ketoprofen ethyl ester in A348V occupies a similar

430 position to the active site, but the methyl substituent at the chiral center of $(S)$-ketoprofen ethyl

431 ester does not make a $\pi$-alkyl interaction with the aromatic pocket formed by F125, and Y170

432 (Figure 3D). Instead, this methyl group make $\pi$-alkyl interactions with residues Y123 and H261. 
433 We assume that, due to the unfavorable orientation of $(S)$-ketoprofen ethyl ester in A348V, the

434 mutant shows no preference for the $S$-enantiomer.

A

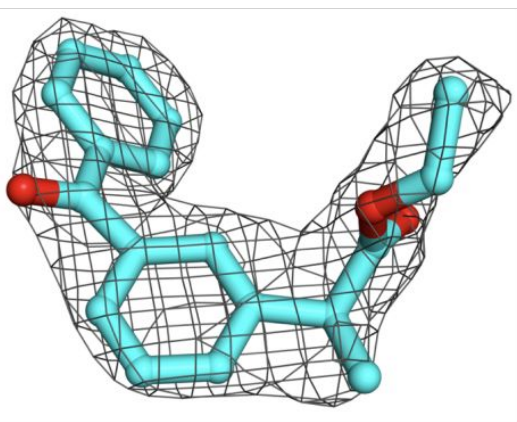

C

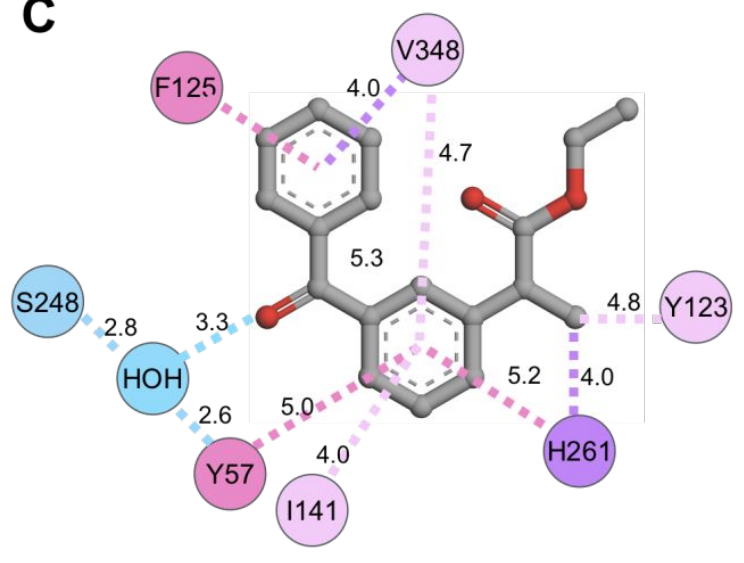

$\square$-Alkyl
$\square$ Water Hydrogen Bond

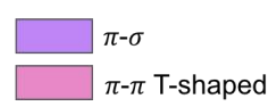

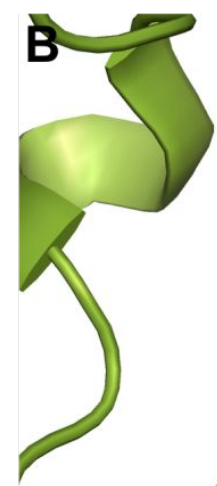

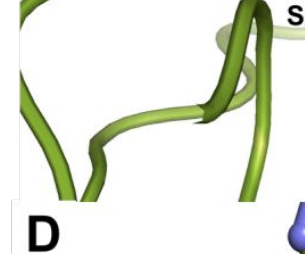

S248
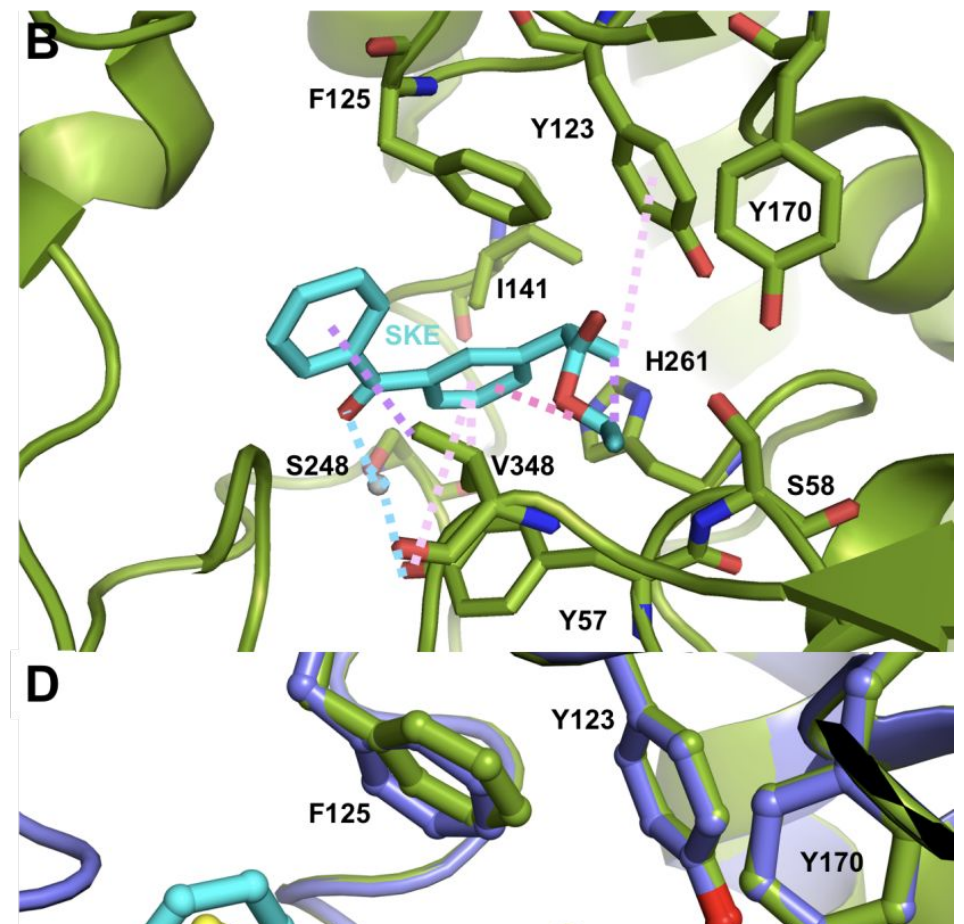

F125

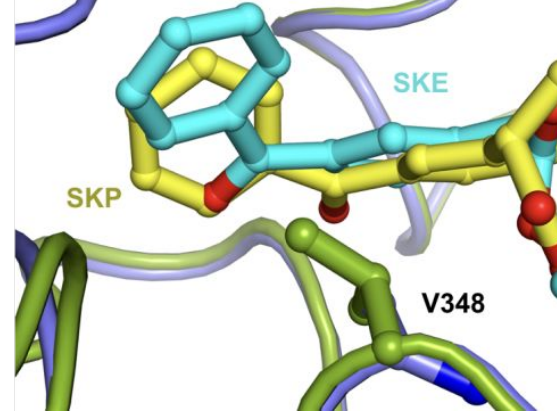

H261
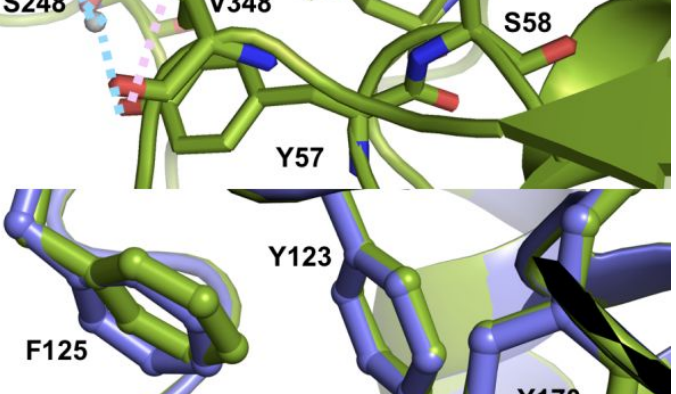

1

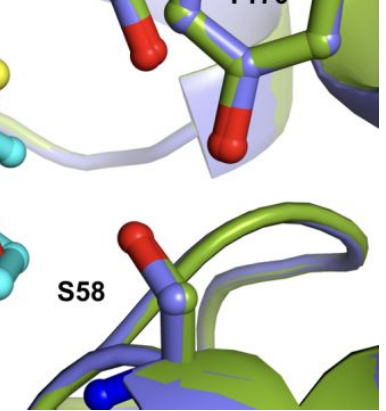

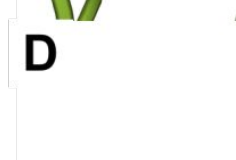

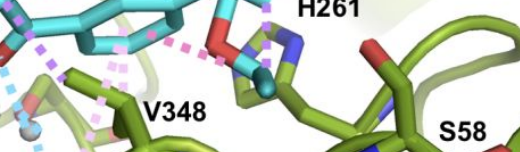

436 Figure 3. Structure of the Mutant Est-Y29 (A348V) Bound to (S)-Ketoprofen Ethyl Ester

437 (A) $2 \mathrm{~F}_{\mathrm{O}}-\mathrm{F}_{\mathrm{C}}$ map contoured at $1.0 \sigma$ shows clear electron density for $(S)$-ketoprofen ethyl ester, 438 shown in cyan in the stick representation. (B) Detailed interactions of $(S)$-ketoprofen ethyl ester 439 (cyan sticks) and residues in the active site of the mutant Est-Y29, A348V (split pea stick 440 representation). The water molecule involved in SKP binding is shown as grey sphere. 441 Hydrogen bonds are indicated with green dashed lines. $\pi$ interactions $(\pi-\sigma, \pi-\pi$, and $\pi$-alkyl) 442 are shown with violet dashed lines. (C) 2D ligand interaction diagram of (S)-ketoprofen ethyl 443 ester with the active sites of the mutant A348V. Atomic interactions are indicated by the dotted 444 lines and their distances in angstroms are shown. (D) Superimposition of the residues in the 445 active sites of Est-Y29-SKP (slate sticks) and A348V-SKE (split pea sticks) shows the 446 difference of orientation of the methyl group at the chiral center. $(S)$-ketoprofen is shown with 447 yellow sticks while $(S)$-ketoprofen ethyl ester is shown as cyan sticks. 
In an attempt to explain the increased enantioselectivity of the mutant Est-Y29 (F125W), we

tried to solve the structure of the mutant protein with $(S)$-ketoprofen ethyl ester. However, despite extensive efforts, we failed to obtain the complex structure. We therefore crystallized the apo-mutant Est-Y29 F125W in order to examine the aromatic pocket. In this structure, we observed the different conformation of the loop 225-230, which contains two residues R225 and L227 responsible for interacting with the ketoprofen substrates (Figure 4A). In the apoEst-Y29, this loop is missing, possibly due to its flexible nature. Since R225 and L227 contribute to the ligand binding, loop 225-230 becomes ordered when the ligand is introduced, as seen in the structure Est-Y29-SKP and A348V-SKE. In the crystal structure of Est-Y29 F125W, although there is no ligand, R225 makes a strong $\pi$-cation interaction with W125. Therefore, loop 225-230 is still ordered even in the absence of ligand (Figure 4A). However, residues 225-227 are located close to the product or substrate binding site in the Est-Y29-SKP or A348V-SKE.

We also noticed that residue W125 of F125W shows similar orientation to F125 of Est-

Y29-SKP (Figure 4B). For the quantitative analysis of the substrate binding sites in the wildtype and F125W, their cavity volumes were calculated. The results showed that volumes of the wild-type and F125W Est-Y29 are $1240 \mathrm{~A}^{3}$, and $582 \mathrm{~A}^{3}$, respectively. The difference in the volume of each structure is mostly due to the structural change in the loop (R225-L230) (Figure

4). For example, in the case of F125W, the residues 225-227 occupied the substrate binding sites; accordingly, the volume of active site cavity is the smallest. For the comparison between the wild-type and F125W mutant, we made a model of F125W by changing F125 into tryptophan residue followed by energy minimization, using the wild-type structure as a 472 template. The cavity volume of this model is $711 \mathrm{~A}^{3}, 40 \%$ less than the volume of the wildtype Est-Y29. The volume in the mutant cavity is reduced because W125 closes the hole to the 
smaller pocket (Figure. S4).). Although the cavity volume has been changed, it is noteworthy

that the size of the substrate binding pocket seems to be not changed since the smaller pocket

is not involved in the substrate binding, suggesting that substrate binding is not affected by the

mutation at F125. From this analysis, it is expected that the area of the hydrophobic surface

that interacts with the alkyl moiety of substrate has been increased in F125W since the area

corresponding to the door of the hole to the extra-pocket is covered by the tryptophan residue

(Figure S4), which implies that F125W might have the enhanced binding affinity to the substrate in comparison to the wild-type enzyme.

482

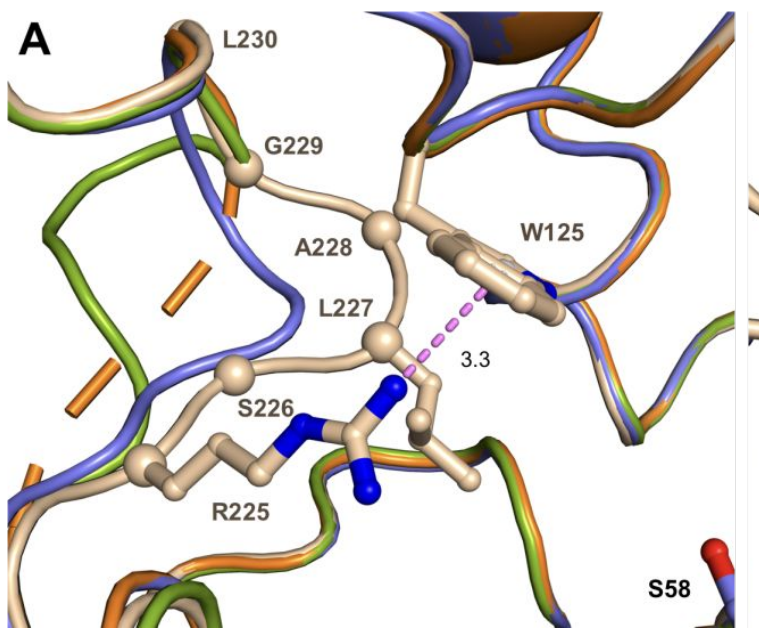

\section{B}
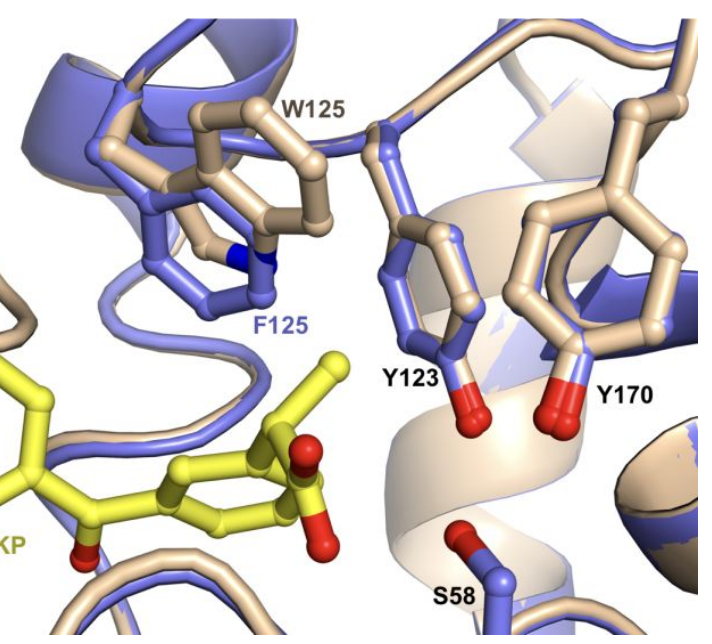

Figure 4. Superimposed Active Site Structures of Est-Y29

(A) Superimposition of apo Est-Y29 (orange cartoon), Est-Y29-SKP (slate cartoon), Est-Y29

A348V-SKE (split pea cartoon), and Est-Y29 F125W (wheat cartoon) show multiple conformations of the loop 225-230. Residues from 225 to 230 are shown as spheres. The $\pi$ cation interaction between R225 and W125 in Est-Y29 F125W is shown as violet dashed lines. Distances in angstroms between the center of aromatic ring of W125 and a guanidinyl group of R225 is shown. (B) Superimposition of residues in the active sites of Est-Y29-SKP (slate sticks) and Est-Y29 F125W (wheat sticks) shows that the aromatic pocket formed by Y123, W125, and Y170 in Est-Y29 F125W is commonly conserved in the wild-type and F125W EstY29 structures. $(S)$-ketoprofen of Est-Y29-SKP is shown as yellow sticks. 
496 To understand the enhanced enantioselectivity of the mutant F125W, we measured enzyme

497 kinetic parameters of the wild-type and F125W EST-Y29 with two substrates $(R)$ - and $(S)$ 498 ketoprofen ethyl ester (Figure 5 and Table S1). It can be seen that the catalytic efficiency ( $k_{\text {cat }}$ $\left.499 / K_{\mathrm{M}}\right)$ of F125W was 4-fold higher for $(S)$-ketoprofen ethyl ester than for $(R)$-ketoprofen ethyl 500 ester, which is improved from 1.7-fold higher catalytic efficiency for $(S)$-ketoprofen ethyl ester 501 in the wild-type enzyme. Moreover, the catalytic efficiency of F125W for $(S)$-ketoprofen ethyl 502 ester was in fact improved 1.4-fold from the wild-type enzyme while the catalytic efficiency 503 of F125W for (R)-ketoprofen ethyl ester was worsened 1.8-fold from that of the wild-type Est504 Y29. This clearly shows that the enhanced enantioselectivity actually comes with the 505 comparable or enhanced catalytic efficiency for the $(S)$-enantiomer as well as the significantly 506 decreased catalytic efficiency for the $(R)$-enantiomer. 507

508
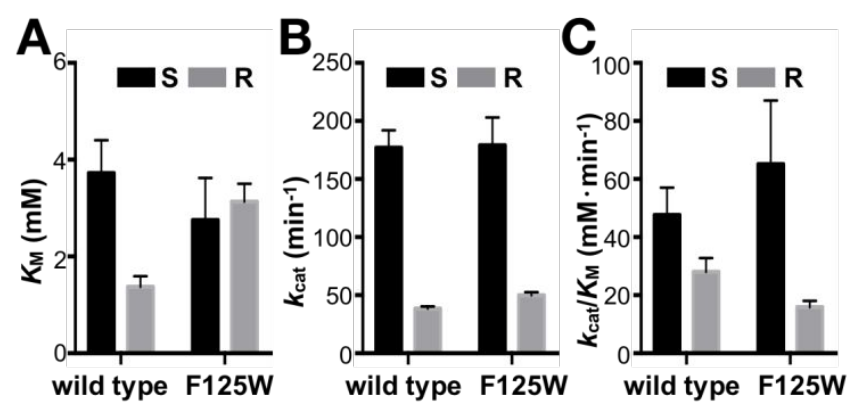

509 Figure 5. Kinetic Analysis of the Wild-Type and F125W Est-Y29 to (R)- and (S)510 Ketoprofen Ethyl Esters. (A) $K_{\mathrm{M}}$ of the wild-type and F125W Est-Y29 to $(S)$-ketoprofen 511 ethyl ester (black bar) and ( $R$ )-ketoprofen ethyl ester (gray bar). (B) $k_{\text {cat }}$ of the wild-type and 512 F125W to $(S)$-ketoprofen ethyl ester (black bar) and ( $R$ )-ketoprofen ethyl ester (gray bar). (C) 513 Catalytic efficiency $k_{\text {cat }} / K_{\mathrm{M}}$ of the wild-type and F125W Est-Y29 to $(S)$-ketoprofen ethyl ester 514 (black bar) and $(R)$-ketoprofen ethyl ester (gray bar). For fitting on Michaelis-Menten equation, 515 5-8 points of substrate concentrations were used. Catalytic efficiency was obtained from 516 division of $k_{\text {cat }}$ by $K_{\mathrm{M}}$. Graphs were drawn using the values in Table S1. 


\section{$518 \quad 3.7$ Functional Improvement of Engineered Est-Y29}

519 The wild-type Est-Y29 presented high thermostability with marginal enantioselectivity, which

520 is an attractive characteristic for industrial application ${ }^{23}$. In this study, we improve the 521 enantioselectivity of Est-Y29 by mutating Phe at $125^{\text {th }}$ residue to Trp by five-fold increasing 522 the $E$ value toward the $(S)$-ketoprofen ethyl ester compared with the wild-type enzyme. For 523 industrial applications, it is necessary to test the stability and hydrolysis activity of the mutant 524 in comparison with the wild-type enzyme. The $\mathrm{T}_{\mathrm{m}}$ values of the wild-type and the mutant are 525 quite similar (344.6 K for the wild-type and 345.6 K for the mutant F125W), suggesting that 526 replacement of F125 to W125 does not alter the protein stability (Figure 6A). However, mutant 527 F125W showed lower residual activity than the wild-type protein after incubation at higher 528 temperature conditions (Figure 6B). Therefore, we further investigated whether the substrate enantiomers did not contribute the stability of enzymes since there was little Tm value change (Figure S5). Therefore, it is very likely that the lower activity of F125W at high temperature is caused not by the lower complex stability but by the lower catalytic activity.

When the Roche ester enantiomer was applied to characterize enantioselectivity of wild-type and mutant Est-Y29 (Figure 6C), we found that the residual enantioselectivity of mutant Est-Y29 did not change at high temperatures (Figure 6D). The ratio of activity to $S$ 537 and $R$-form is sustained in overall temperature up to $338 \mathrm{~K}$. Interestingly, unlike the ketoprofen 538 ethyl ester, both activity and selectivity to the $S$-enantiomer of the wild-type Est-Y29 were 539 higher than those of the mutant F125W when the Roche ester was used as a substrate. This 540 result can be explained by that $\mathrm{F} 125 \mathrm{~W}$ is designed to fit on $(S)$-ketoprofen ethyl ester by 541 strengthened aromatic environment at benzophenone group and chiral center. Thus, the 542 mutation is not fatal and does not destroy intramolecular interactions, pocket structure, and 
543 selective function, but it does increase its targeted enantioselectivity to $S$-enantiomer of 544 ketoprofen ethyl ester.

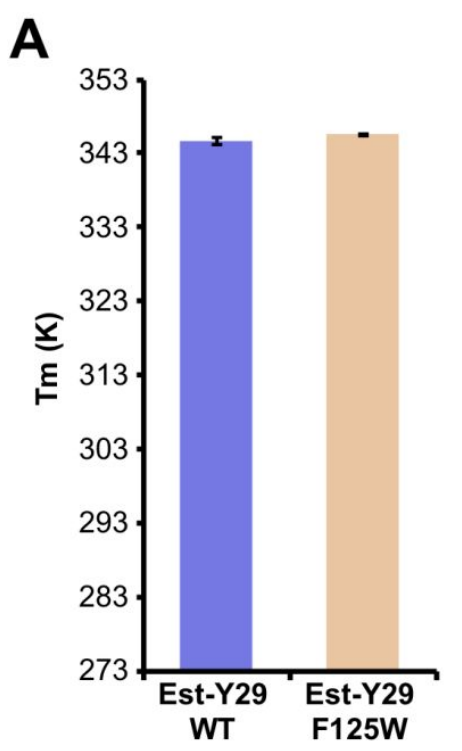

B

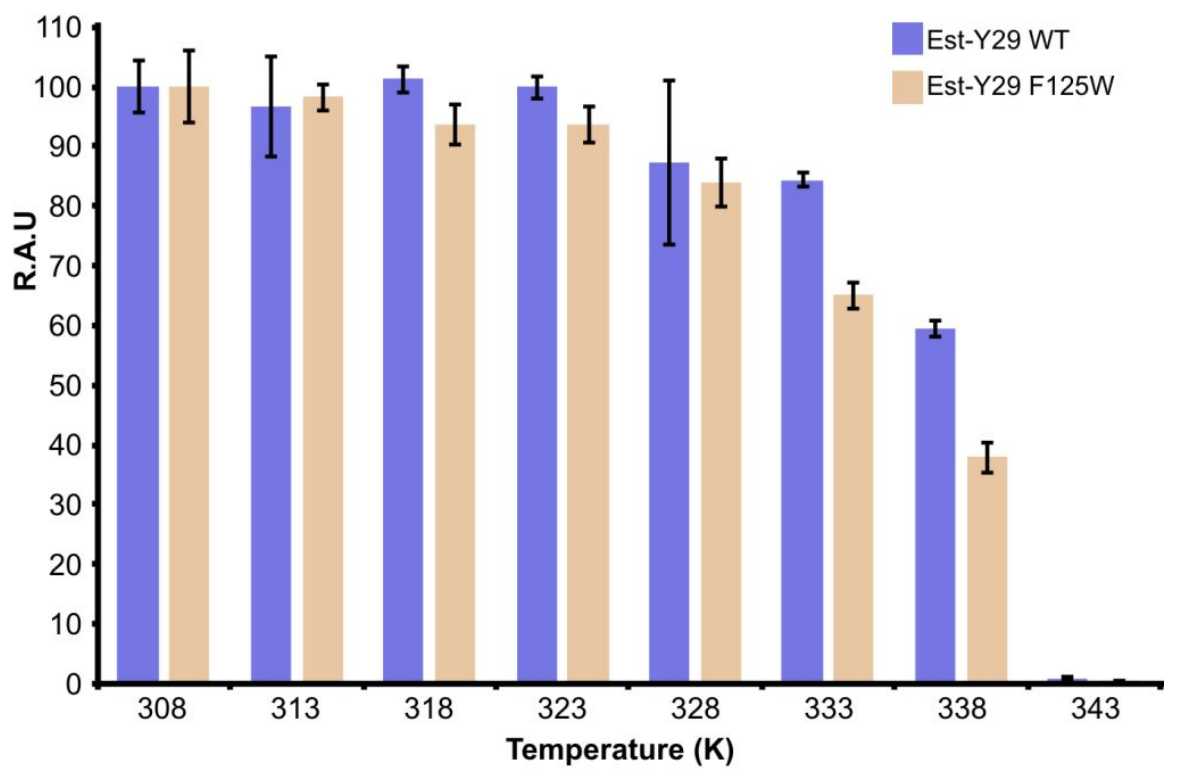<smiles>COC(=O)[C@H](C)CO</smiles>

(S)-Roche ester<smiles>COC(=O)[C@H](C)CO</smiles>

(R)-Roche ester

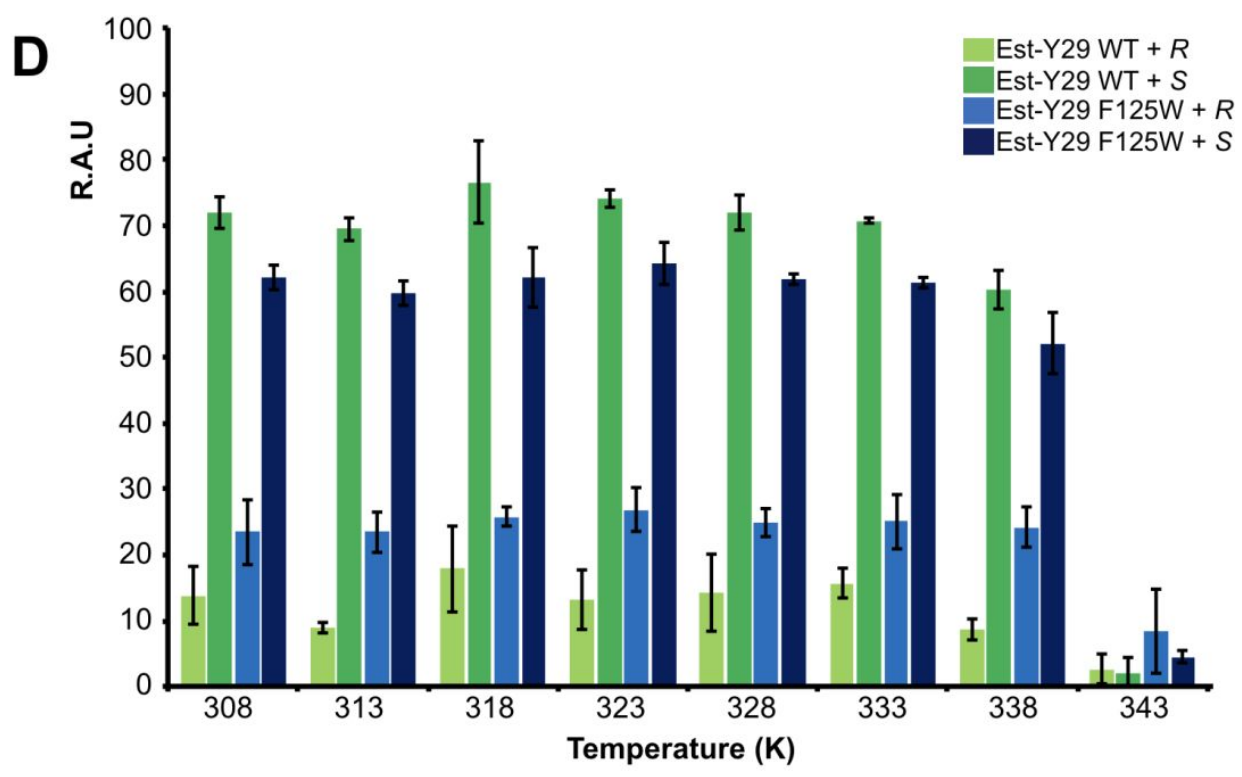

Figure 3. Thermal Stability and Enantioselectivity of the Wild-Type and F125W Est-Y29.

547 (A) Thermal melting points of the wild-type (Est-Y29 WT) and mutant Est-Y29 (Est-Y29

548 F125W) obtained by circular dichroism (CD) analysis. (B) Residual activity of the wild-type

549 and F125W Est-Y29 at different temperature conditions. (C) Chemical structures of $(R)$ - and

550 (S)-Roche ester used in the enantioselectivity assay in (D). (D) Temperature dependent change

551 in the enantioselectivity of the wild-type and F125W Est-Y29. 


\section{4. Discussion}

556 In this study, we solved the crystal structures of the thermostable Est-Y29 in the product-

557 bound form and its mutant in substrate-bound form. From the structural analysis, combining 558 with docking studies of Est-Y29 with both $(R)$ - and $(S)$-ketoprofen ethyl ester, we explained 559 the molecular basis of enantioselectivity toward the $(S)$-ketoprofen ethyl ester. We found that $560 \mathrm{Y} 123, \mathrm{~F} 125$, and Y170 create an aromatic pocket that is involved in the $\pi$-alkyl interaction with 561 the methyl substituent of (S)-ketoprofen. Accordingly, the mutants F125W showed higher 562 enantioselectivity with five-fold enhanced enantioselectivity compared to the wild-type Est563 Y29 due to the increased $\pi$-interaction.

In addition, by kinetic analyses, we showed that F125W mutation decreased $K_{\mathrm{M}}$ for $(S)$ ketoprofen ethyl ester 1.3-fold, in comparison to the wild-type enzyme, while the same mutation increased $K_{\mathrm{M}}$ 1.8-fold for $(R)$-ketoprofen ethyl ester. This suggests the $\mathrm{F} 125 \mathrm{~W}$ mutation created a higher substrate binding affinity for $(S)$-ketoprofen ethyl ester, while it decreased the binding affinity for $(R)$-ketoprofen ethyl ester compared to the wild-type EstY29. On the other hand, the wild-type Est-Y29 and F125W showed similar values of $k_{\text {cat }}$. These changes led to a 4-fold higher catalytic efficiency $\left(k\right.$ cat $\left./ K_{\mathrm{M}}\right)$ of F125W for $(S)$-ketoprofen ethyl ester than for $(R)$-ketoprofen ethyl ester. Hence, it is highly likely that the changes in binding affinity, mainly the decreased binding affinity for R-substrate, is a major driving force in the higher enantioselectivity of F125W in favor of the S-form ketoprofen ester. Taking these together, we can propose that the enantioselectivity of Est-Y29 comes from the difference in binding affinity rather than the transition state structure that is represented by the $k_{\text {cat }}$ value. to the $(S)$-conformer since the docking scores of $(S)$-ketoprofen ethyl ester bound to the mutant 
579 higher in the case of the wild-type Est-Y29 (Table S2). In the structural aspect, this binding

580 affinity change can be explained by the $\pi$-interaction change. Superposition of docking models

581 of $(R)$ - and $(S)$-ketoprofen ethyl ester substrates bound to Est-Y29 revealed that the first priority

582 group $\left(\mathrm{COOC}_{2} \mathrm{H}_{5}\right)$ and the second priority group (PheCOPhe) in the chiral center are positioned

583 in a similar space, but the position of remaining substituents $\mathrm{CH}_{3}$ and $\mathrm{H}$ are exchanged (Figure

584 7). For faster reacting $(S)$-ketoprofen ethyl ester, the substituent $\mathrm{CH}_{3}$ lies close to the aromatic

585 pocket formed by $\mathrm{Y} 123, \mathrm{~F} 125$, and $\mathrm{Y} 170$, and the substituent $\mathrm{H}$ is closed to the side chain of

586 F125 and A348. However, the substituent $\mathrm{H}$ is located in the aromatic pocket in the slower-

587 reacting $(R)$-ketoprofen ethyl ester structure, creating two mismatches (Figure $7 \mathrm{~B})$. Docking

588 models of F125W to $(S)$-ketoprofen ethyl ester (F125W-SKE) and (R)-ketoprofen ethyl ester

589 (F125W-SKE) also support this view (Figure S6). Although the docking models of F125W are

590 not identical to those of the wild-type enzyme, the closer distance of a benzophenone ring and

591 chiral center in $(S)$-ketoprofen ethyl ester to W125 than those in $(R)$-ketoprofen ethyl ester is

592 consistently found in the docking models of the wild type Est-Y29 (Figure 2). Trp was found

593 as the greatest enrichment in the carbohydrate-binding pockets. Electron-rich aromatic Trp is

594 the most likely residue to engage in the $\mathrm{CH}-\pi$ interactions ${ }^{41}$, which enhances the $(S)$-ketoprofen

595 ethyl ester-binding to Est-Y29 by increased binding affinity between the pocket and methyl

596 group in the substrate. 


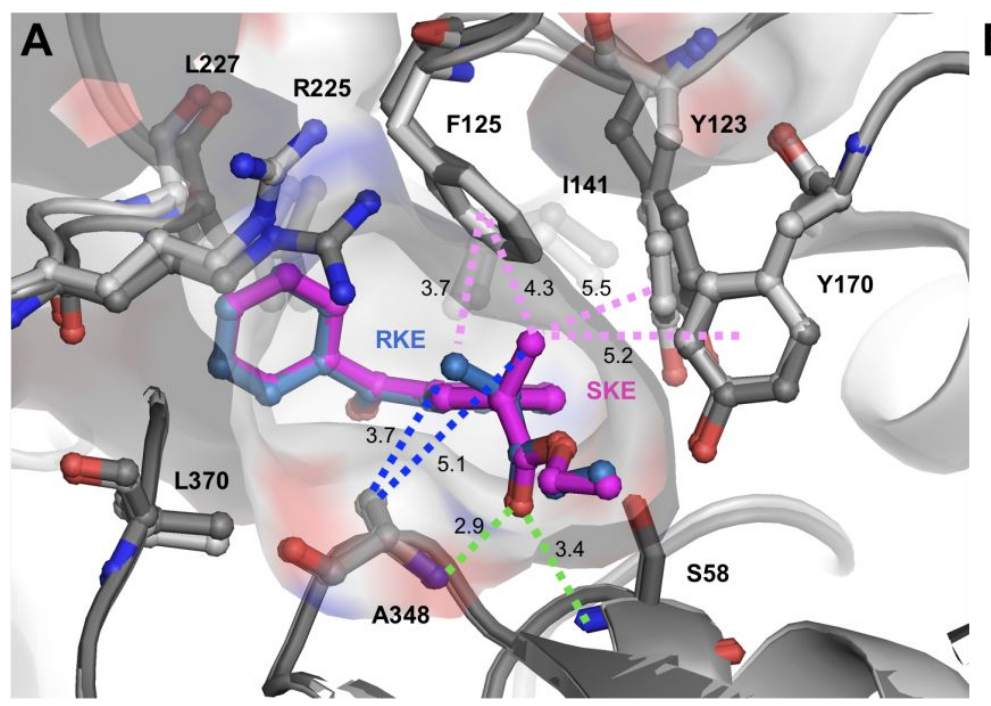

\section{B}

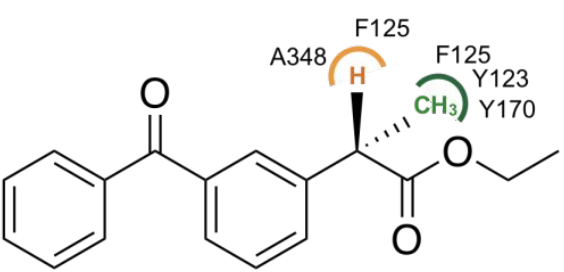

(S)-ketoprofen ethyl ester: Fast enantiomer

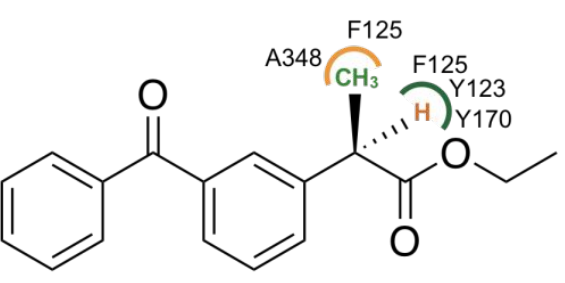

$(R)$-ketoprofen ethyl ester: Slow enantiomer

598 599

Figure 4. Structural Basis of Enantioselectivity of Est-Y29 toward (S)-Ketoprofen Ethyl

\section{Ester}

(A) Superimposition of the docking models of $(R)$ - and $(S)$-ketoprofen ethyl ester. $(S)$ ketoprofen ethyl ester is shown as magenta sticks while $(R)$-ketoprofen ethyl ester is shown as marine sticks. Important residues of Est-Y29 are shown as grey stick representation. Hydrogen bonds are indicated with green dashed lines. $\pi$-interactions ( $\pi-\pi$ and $\pi$-alkyl) are shown with violet dashed lines. $(R)$-ketoprofen ethyl ester and $(S)$-ketoprofen ethyl ester are marked as RKE and SKE, respectively. (B) Schematic of racemic $(R, S)$-ketoprofen ethyl ester in the active site of Est-Y29 in two possible configurations. The enantioselectivity originates from the different orientation of two substituents $\left(\mathrm{H}\right.$ and $\left.\mathrm{CH}_{3}\right)$ at the stereo-center of the ligands. The binding of (S)-ketoprofen ethyl ester is preferred due to the stronger $\pi$-alkyl interaction of the methyl group of (S)-ketoprofen ethyl ester to the aromatic pocket formed by Y123, F125, and Y170.

To enhance enzyme activity to a specific enantiomer, residues around substrate-binding site are regarded as effective targets. Especially, for enantioselectivity, the chiral centerpositioned space in enzyme-substrate complex structures is an attractive area to regulate specificity to each enantiomer. By interfering with binding to the pocket and forming tetrahedral intermediates of unfavorable enantiomers, the enzyme allows desired enantiomers to approach and process them. To that end, we prepared A348V in which the V348 residue was 
618

619

620

621

622

623

624

625

626

627

628

629

630

631

632

633

634

635

636

637

638

639 Associated Content

640

641

\section{Supporting Information}

expected to clash with methyl substituent to chiral carbon in $(R)$-ketoprofen ethyl ester and repel it from the pocket. However, the replaced V348 forms additional $\pi$-alkyl interaction with the common benzophenone group in both the $R$ - and $S$-enantiomers (Figure 3B), and increases especially the $R$-enantiomer binding by hydrophobic interaction instead of expected hindrance to the $R$-form. Moreover, distortion of ligand binding in A348V induced loss of interaction with residues for the oxyanion hole, and reduced stability of A348V to $(S)$-ketoprofen ethyl ester (Figure 4C). These were coincident with results of enantiomer analysis using HPLC as increase of activity to $R$-enantiomer and almost equal activity in both forms (Table 3 ).

In kinetic resolution, enantioselectivity of enzymes is also influenced by solution and temperature ${ }^{42}$. The racemic temperature $\left(T_{r}\right)$ is a conceptual temperature, which makes enzymes produce both enantiomers equally at the $\mathrm{T}_{\mathrm{r}}{ }^{43}$. Jin et al. showed the temperature effect on enantioselectivity and increase or decrease of temperature from the $T_{r}$ affected enantioselectivity ${ }^{44}$. Solution components such as detergent are also critical to determining $E$ value $^{45}$. In this study, we set the temperature to $323 \mathrm{~K}$ for selective hydrolysis in a fixed buffer. To improve enantioselectivity, the characterization of optimal conditions such as buffer and reaction temperature is additionally required to magnify endogenous enzyme selectivity. Therefore, we expect the enantioselectivity of F125W could be further enhanced by optimizing the reaction conditions, which is necessary for further industrial application of this enzyme. Furthermore, if F125W is used as a template for the directed evolution, Est-Y29 is expected to be engineered as a biocatalyst suitable for the industrial production of $(S)$-ketoprofen ${ }^{464641}$

Substrate and product analyses data and docking model description (.pdf) 
642 Crystallographic data (.cif) of Est-Y29-SKP (PDB code: 5ZWR), Est-Y29 F125W (PDB code: 643 5ZWV) and Est-Y29 A348V-SKE (PDB code: 5ZWQ)

644 This information is available free of charge on the ACS Publications website.

645

646 Author Information

647 ORCID

648 Tri Duc Ngo: 0000-0002-9599-0654

649 Changsuk Oh: 0000-0001-6347-1609

650 Kyeong Kyu Kim: 0000-0003-2515-8894

651

652 Corresponding Author

653 *To whom all correspondence should be addressed: Tel: 82-31-299-6136, Fax: 82-31-299654 6159, E-mail: kyeongkyu@skku.edu

655

656 Author Contribution

657 \$ These authors equally contribute this work.

658 KKK conceived and supervised the work. KKK, TDN, CO, WY, YR contributed to data 659 interpretation. TDN, CO solved the crystal structure. TND, MP, CS performed the docking 660 analysis, TDN, CO, PM, SL, KP, LN, HB, SBK, SY, BHR performed biochemical 661 experiments. KKK, TDN, CO wrote the manuscript. All authors reviewed the final 662 manuscript.

663

664 Funding Sources

665 This study was funded by National Research Foundation of Korea grants 666 (2017M3A9E4078553) and the Next-Generation BioGreen 21 Program (SSAC-PJ01317301). 


\section{References}

669 1. Sheldon, R. A.; Woodley, J. M., Role of Biocatalysis in Sustainable Chemistry. Chem $670 \quad \operatorname{Rev} 2018,118,801-38$.

$6712 . \quad$ Bommarius, A. S.; Blum, J. K.; Abrahamson, M. J., Status of Protein Engineering for 672 Biocatalysts: How to Design an Industrially Useful Biocatalyst. Curr Opin Chem Biol 2011, $67315,194-200$

674 3. Bornscheuer, U. T.; Huisman, G. W.; Kazlauskas, R. J.; Lutz, S.; Moore, J. C.; Robins, 675 K., Engineering the Third Wave of Biocatalysis. Nature 2012, 485, 185-94.

676 4. Bordes, F.; Cambon, E.; Dossat-Letisse, V.; Andre, I.; Croux, C.; Nicaud, J. M.; Marty, 677 A., Improvement of Yarrowia Lipolytica Lipase Enantioselectivity by Using Mutagenesis 678 Targeted to the Substrate Binding Site. Chembiochem 2009, 10, 1705-13.

679 5. Jaeger, K. E.; Reetz, M. T., Microbial Lipases Form Versatile Tools for Biotechnology. 680 Trends Biotechnol 1998, 16, 396-403.

681 6. Reetz, M. T.; Bocola, M.; Wang, L. W.; Sanchis, J.; Cronin, A.; Arand, M.; Zou, J.; 682 Archelas, A.; Bottalla, A. L.; Naworyta, A.; Mowbray, S. L., Directed Evolution of an 683 Enantioselective Epoxide Hydrolase: Uncovering the Source of Enantioselectivity at Each 684 Evolutionary Stage. J Am Chem Soc 2009, 131, 7334-43.

$6857 . \quad$ Reetz, M. T.; Jaeger, K. E., Enantioselective Enzymes for Organic Synthesis Created 686 by Directed Evolution. Chemistry 2000, 6, 407-12.

687 8. Reetz, M. T.; Wu, S., Laboratory Evolution of Robust and Enantioselective Baeyer688 Villiger Monooxygenases for Asymmetric Catalysis. J Am Chem Soc 2009, 131, 15424-32.

689 9. Turner, N. J., Directed Evolution Drives the Next Generation of Biocatalysts. Nat Chem 690 Biol 2009, 5, 567-73. 
691 10. Ema, T.; Fujii, T.; Ozaki, M.; Korenaga, T.; Sakai, T., Rational Control of 692 Enantioselectivity of Lipase by Site-Directed Mutagenesis Based on the Mechanism. Chem 693 Commun (Camb) 2005, 4650-1.

694 11. Ema, T.; Kamata, S.; Takeda, M.; Nakano, Y.; Sakai, T., Rational Creation of Mutant 695 Enzyme Showing Remarkable Enhancement of Catalytic Activity and Enantioselectivity 696 toward Poor Substrates. Chem Commun (Camb) 2010, 46, 5440-2.

697 12. Magnusson, A. O.; Takwa, M.; Hamberg, A.; Hult, K., An S-Selective Lipase Was 698 Created by Rational Redesign and the Enantioselectivity Increased with Temperature. Angew 699 Chem Int Ed Engl 2005, 44, 4582-5.

700 13. Henke, E.; Bornscheuer, U. T.; Schmid, R. D.; Pleiss, J., A Molecular Mechanism of 701 Enantiorecognition of Tertiary Alcohols by Carboxylesterases. Chembiochem 2003, 4, 485-93. 702 14. Harris, R. H.; Vavra, I., Ketoprofen. In Rainsford, D. K. (Ed.), Anti-Inflammatory and 703 Anti-Rheumatic Drugs: Inflammation Mechanisms and Actions of Traditional Drugs. CRC 704 Press: Boca Raton, 1985; Vol. 2, p 151-69.

705 15. Hayball, P. J., Chirality and Nonsteroidal Anti-Inflammatory Drugs. Drugs 1996, 52 706 Suppl 5, 47-58.

707 16. Carabaza, A.; Cabre, F.; Rotllan, E.; Gomez, M.; Gutierrez, M.; Garcia, M. L.; Mauleon, 708 D., Stereoselective Inhibition of Inducible Cyclooxygenase by Chiral Nonsteroidal 709 Antiinflammatory Drugs. J Clin Pharmacol 1996, 36, 505-12.

710 17. Ghezzi, P.; Melillo, G.; Meazza, C.; Sacco, S.; Pellegrini, L.; Asti, C.; Porzio, S.; 711 Marullo, A.; Sabbatini, V.; Caselli, G.; Bertini, R., Differential Contribution of R and S Isomers 712 in Ketoprofen Anti-Inflammatory Activity: Role of Cytokine Modulation. J Pharmacol Exp 713 Ther 1998, 287, 969-74.

714 18. Ossipov, M. H.; Jerussi, T. P.; Ren, K.; Sun, H.; Porreca, F., Differential Effects of 715 Spinal $(R)$-Ketoprofen and $(S)$-Ketoprofen against Signs of Neuropathic Pain and Tonic 
716 Nociception: Evidence for a Novel Mechanism of Action of (R)-Ketoprofen against Tactile 717 Allodynia. Pain 2000, 87, 193-9.

718 19. Thayer, A. M., Chemical and Engineering News. Biocatalyst: Houston, 2001.

719 20. U. S. Food and Drug Administration, FDA's Policy Statement for the Development of 720 New Stereoisomeric Drugs. Chirality, 1992; 4, 338-40.

721 21. Tomaszewski, J.; Rumore, M. M., Stereoisomeric Drugs: FDA's Policy Statement and 722 the Impact on Drug Development. Drug Development and Industrial Pharmacy 1994, 20, 119 72339.

724 22. Ong, A. L.; Kamaruddin, A. H.; Bhatia, S., Current Technologies for the Production of 725 (S)-Ketoprofen: Process Perspective. Process Biochemistry 2005, 40, 3526-35.

726 23. Yoon, S.; Kim, S.; Ryu, Y.; Kim, T. D., Identification and Characterization of a Novel 727 (S)-Ketoprofen-Specific Esterase. Int J Biol Macromol 2007, 41, 1-7.

728 24. Kim, S.; Joo, S.; Yoon, S.; Kim, S.; Moon, J.; Ryu, Y.; Kim, K. K.; Kim, T. D., 729 Purification, Crystallization and Preliminary Crystallographic Analysis of Est-Y29: A Novel 730 Oligomeric Beta-Lactamase. Acta Crystallogr Sect F Struct Biol Cryst Commun 2009, 65, 3107312.

732 25. Ngo, T. D.; Ryu, B. H.; Ju, H.; Jang, E. J.; Kim, K. K.; Kim, T. D., Crystallographic 733 Analysis and Biochemical Applications of a Novel Penicillin-Binding Protein/Beta-Lactamase 734 Homologue from a Metagenomic Library. Acta Crystallogr D Biol Crystallogr 2014, 70, 245573566.

736 26. Jin, J. N.; Lee, S. H.; Lee, S. B., Enzymatic Production of Enantiopure Ketoprofen in a 737 Solvent-Free Two-Phase System. Journal of Molecular Catalysis B: Enzymatic 2003, 26, 20973816.

739 27. Otwinowski, Z.; Minor, W., Processing of X-Ray Diffraction Data Collected in 740 Oscillation Mode. Macromolecular Crystallography, Pt A 1997, 276, 307-26. 
741 28. Vagin, A.; Teplyakov, A., Molecular Replacement with Molrep. Acta Crystallogr D $742 \quad 2010,66,22-5$.

743 29. Winn, M. D.; Murshudov, G. N.; Papiz, M. Z., Macromolecular Tls Refinement in 744 Refmac at Moderate Resolutions. Methods Enzymol 2003, 374, 300-21.

745 30. Steiner, R. A.; Lebedev, A. A.; Murshudov, G. N., Fisher's Information in Maximum746 Likelihood Macromolecular Crystallographic Refinement. Acta Crystallogr D Biol Crystallogr $747 \quad 2003,59,2114-24$.

748 31. Adams, P. D.; Afonine, P. V.; Bunkoczi, G.; Chen, V. B.; Davis, I. W.; Echols, N.; 749 Headd, J. J.; Hung, L. W.; Kapral, G. J.; Grosse-Kunstleve, R. W.; McCoy, A. J.; Moriarty, N. 750 W.; Oeffner, R.; Read, R. J.; Richardson, D. C.; Richardson, J. S.; Terwilliger, T. C.; Zwart, P. 751 H., Phenix: A Comprehensive Python-Based System for Macromolecular Structure Solution. 752 Acta Crystallogr D 2010, 66, 213-21.

753 32. Laskowski, R. A.; MacArthur, M. W.; Moss, D. S.; Thornton, J. M., Procheck: A 754 Program to Check the Stereochemical Quality of Protein Structures. Journal of Applied 755 Crystallography 1993, 26, 283-91.

756 33. Tian, W.; Chen, C.; Lei, X.; Zhao, J.; Liang, J., Castp 3.0: Computed Atlas of Surface 757 Topography of Proteins. Nucleic Acids Res 2018, 46, W363-W7.

758 34. Heo, L.; Park, H.; Seok, C., Galaxyrefine: Protein Structure Refinement Driven by 759 Side-Chain Repacking. Nucleic Acids Res 2013, 41, W384-8.

760 35. Sanner, M. F., Python: A Programming Language for Software Integration and 761 Development. J Mol Graph Model 1999, 17, 57-61.

762 36. Trott, O.; Olson, A. J., Software News and Update Autodock Vina: Improving the 763 Speed and Accuracy of Docking with a New Scoring Function, Efficient Optimization, and 764 Multithreading. J Comput Chem 2010, 31, 455-61. 
765 37. Fuhrmann, J.; Rurainski, A.; Lenhof, H. P.; Neumann, D., A New Lamarckian Genetic 766 Algorithm for Flexible Ligand-Receptor Docking. J Comput Chem 2010, 31, 1911-8.

767 38. Lee, G. R.; Heo, L.; Seok, C., Effective Protein Model Structure Refinement by Loop 768 Modeling and Overall Relaxation. Proteins 2016, 84 Suppl 1, 293-301.

769 39. Baek, M.; Shin, W. H.; Chung, H. W.; Seok, C., Galaxydock Bp2 Score: A Hybrid 770 Scoring Function for Accurate Protein-Ligand Docking. J Comput Aided Mol Des 2017, 31, $771 \quad 653-66$.

772 40. Lee, G. R.; Seok, C., Galaxy7tm: Flexible Gpcr-Ligand Docking by Structure 773 Refinement. Nucleic Acids Res 2016, 44, W502-6.

774 41. Hudson, K. L.; Bartlett, G. J.; Diehl, R. C.; Agirre, J.; Gallagher, T.; Kiessling, L. L.; 775 Woolfson, D. N., Carbohydrate-Aromatic Interactions in Proteins. J Am Chem Soc 2015, 137, $776 \quad 15152-60$.

777 42. Sheldon, R. A.; Pereira, P. C., Biocatalysis Engineering: The Big Picture. Chem Soc $778 \operatorname{Rev} 2017,46,2678-91$.

779 43. Duan, G.; Chen, J. Y., Racemic Temperature and Stereochemistry. Trends in 780 Biotechnology 1997, 15, 333.

781 44. Jin, X.; Liu, B.; Ni, Z.; Wu, Q.; Lin, X., A Novel Control of Enzymatic 782 Enantioselectivity through the Racemic Temperature Influenced by Reaction Media. Enzyme 783 Microb Technol 2011, 48, 454-7.

784 45. Liu, J.; Zhang, Y.; Qiu, L.; Yang, F.; Ye, L.; Xia, Y., Kinetic Resolution of Ketoprofen 785 Ester Catalyzed by Lipase from a Mutant of Cbs 5791. J Ind Microbiol Biotechnol 2004, 31, $786 \quad 495-9$.

787 46. Kim, J.; Kim, S.; Yoon, S.; Hong, E.; Ryu, Y., Improved Enantioselectivity of 788 Thermostable Esterase from Archaeoglobus Fulgidus toward $(S)$-Ketoprofen Ethyl Ester by 
789 Directed Evolution and Characterization of Mutant Esterases. Appl Microbiol Biotechnol 2015, $79099,6293-301$.

791

792 
793

794 TOC

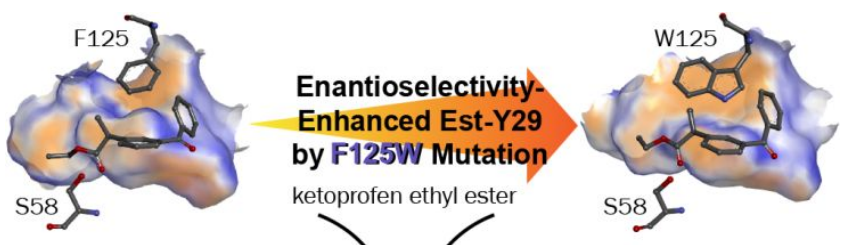

795
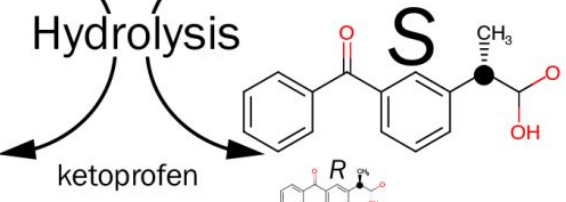